\title{
Lower Spectral Branches of a Particle Coupled to a Bose Field
}

\author{
Nicolae Angelescu ${ }^{1}$, Robert A. Minlos ${ }^{2}$, \\ and \\ Valentin A. Zagrebnov ${ }^{3}$
}

\begin{abstract}
${ }^{1}$ National Institute of Physics and Nuclear Engineering "H. Hulubei", P.O.Box MG-6, Bucharest, Romania, e-mail: nangel@theory.nipne.ro

${ }^{2}$ Institute for Information Transmissions Problems, Bolshoj Karetny per.19, GSP-4, Moscow 101447, Russia, e-mail: minl@ittp.ru

${ }^{3}$ Université de la Méditerranée and Centre de Physique Théorique - Luminy, Case 907, Marseille 13288, Cedex 9, France, e-mail: zagrebnov@cpt.univ-mrs.fr
\end{abstract}

\begin{abstract}
The structure of the lower part (i.e. $\varepsilon$-away below the two-boson threshold) spectrum of Fröhlich's polaron Hamiltonian in the weak coupling regime is obtained in spatial dimension $d \geq 3$. It contains a single polaron branch defined for total momentum $p \in G^{(0)}$, where $G^{(0)} \subset \mathbb{R}^{d}$ is a bounded domain, and, for any $p \in \mathbb{R}^{d}$, a manifold of polaron + one-boson states with boson momentum $q$ in a bounded domain depending on $p$. The polaron becomes unstable and dissolves into the one boson manifold at the boundary of $G^{(0)}$. The dispersion laws and generalized eigenfunctions are calculated.
\end{abstract}

\section{Introduction}

We consider the quantum system consisting of a particle coupled with a Bose field by an interaction linear in the creation-annihilation operators, known in the physics literature as Fröhlich's polaron model [1]. There are many papers, both physical and mathematical, devoted to this subject, see [2]-[5]. These are mainly concerned with the ground state $F_{p}^{(0)}$ of the Hamiltonian $H_{p}$ of the system at fixed total momentum $p$ acting in the Hilbert space $\mathcal{H}(p)$ (see below). It is shown that for sufficiently small particle-field coupling constant $\alpha$ the ground state $F_{p}^{(0)}$ exists only for momentum $p$ in a certain domain $G^{(0)} \subset \mathbb{R}^{d}$, where $G^{(0)}$ is bounded for space dimension $d \geq 3$ and $G^{(0)}=\mathbb{R}^{d}$ for $d=1,2$. The ground state describes the "polaron", i.e. the particle in a "cloud of virtual bosons".

Here we study the next, "one-boson", branch of the spectrum of $H_{p}$ for $d \geq 3$. The expected mathematical picture is the following: there exists an invariant subspace 
$\mathcal{H}_{1}(p) \subset \mathcal{H}(p)$ of the operator $H_{p}$, which is isomorphic in a natural way with $L_{2}\left(G_{p}^{(1)}, d q\right)$, where $G_{p}^{(1)} \subset \mathbb{R}^{d}$ is a certain bounded domain, such that $H_{p}$ acts in this subspace as multiplication with a function $\xi_{p}(q)$, which can be viewed as the energy of a boson of momentum $q$ (while the total momentum of the system is $p$ ). The range of this function is the segment $\left[\lambda_{1}(p), \lambda_{2}(p)\right)$, where $\lambda_{1}(p)$ and $\lambda_{2}(p)$ are the thresholds of the one- and two-boson states, respectively. Moreover, in the subspace orthogonal to $\mathcal{H}_{0}(p) \oplus \mathcal{H}_{1}(p)$, where $\mathcal{H}_{0}(p)=\left\{c F_{p}^{(0)}\right\}$ is the one-dimensional subspace generated by the ground state whenever it exists, the spectrum of $H_{p}$ lies above $\lambda_{2}(p)$ (this latter property will be called "the completeness of the one-boson spectrum"). The states in $\mathcal{H}_{1}(p)$ can be viewed as scattering states of a boson and a polaron.

Unfortunately, we shall obtain here only part of the above picture. Namely, we are able to construct only a subspace $\mathcal{H}_{1}^{\kappa}(p) \subset \mathcal{H}_{1}(p)$ isomorphic to $L^{2}\left(G_{p}^{(1), \kappa}, d q\right)$, where $G_{p}^{(1), \kappa}=\left\{q \in G_{p}^{(1)}: \xi_{p}(q)<\kappa\right\}$. Here, $\kappa<\lambda_{2}(p)$ can be chosen arbitrarily close to $\lambda_{2}(p)$, at the expense of taking the coupling constant $\alpha$ sufficiently small. Apparently, our techniques allow the construction of the whole space $G_{p}^{(1)}$ and the proof of the completeness of the one-boson spectrum for sufficiently large space dimension $d$.

Our analysis of the one-boson branch covers only the cases $d \geq 3$, though we expect that the same picture holds in lower dimension, with $G_{p}^{(1)}=\mathbb{R}^{d}$ for $d=1,2$. The calculations are based on a technique used by one of the authors in [2], and also on certain facts connected with the spectral analysis of the so-called generalized Friedrichs model [6].

We proceed now to a detailed presentation of the model and a precise statement of the main result.

The state space of our model is the Hilbert space

$$
\mathcal{H}=L^{2}\left(\mathbb{R}^{d}\right) \otimes \mathcal{F},
$$

where $\mathcal{F}$ is the symmetric (boson) Fock space

$$
\mathcal{F}=\mathcal{F}_{\text {sym }}\left(L^{2}\left(\mathbb{R}^{d}\right)\right)=\bigoplus_{n=0}^{\infty} \mathcal{H}^{(n)},
$$

with $\mathcal{H}^{(0)}=\mathbb{C}, \mathcal{H}^{(n)}=\left(L^{2}\left(\mathbb{R}^{d}\right)\right)_{\text {sym }}^{\otimes n}$ the symmetric tensor power $(n \geq 1)$. Thus, the vectors of $\mathcal{H}$ are sequences

$$
F=\left\{f_{0}\left(p_{0}\right), f_{1}\left(p_{0} ; q\right), \ldots, f_{n}\left(p_{0} ; q_{1}, \ldots, q_{n}\right), \ldots\right\},
$$

where $f_{n}$ are, for every $p_{0} \in \mathbb{R}^{d}$, symmetric functions of the variables $q_{1}, \ldots, q_{n}$, and the norm is given by

$$
\|F\|^{2}=\int_{\mathbb{R}^{d}}\left|f_{0}\left(p_{0}\right)\right|^{2} d p_{0}+\sum_{n=1}^{\infty} \frac{1}{n !} \int_{\mathbb{R}^{d}} \int_{\mathbb{R}^{n d}}\left|f_{n}\left(p_{0} ; q_{1}, \ldots, q_{n}\right)\right|^{2} d p_{0} \prod_{i=1}^{n} d q_{i} .
$$


The Hamiltonian of our system has the form

$$
H=H_{0}^{\text {part }}+H_{0}^{\text {field }}+\alpha H_{\text {int }},
$$

where $\alpha>0$ is a coupling constant and

$$
\begin{aligned}
\left(H_{0}^{\text {part }} F\right)_{n}\left(p_{0} ; q_{1}, \ldots q_{n}\right) & =\frac{1}{2} p_{0}^{2} f_{n}\left(p_{0} ; q_{1}, \ldots, q_{n}\right) \\
\left(H_{0}^{f i e l d} F\right)_{n}\left(p_{0} ; q_{1}, \ldots q_{n}\right) & =\left(\sum_{i=1}^{n} \varepsilon\left(q_{i}\right)\right) f_{n}\left(p_{0} ; q_{1}, \ldots, q_{n}\right) \\
\left(H_{\text {int }} F\right)_{n}\left(p_{0} ; q_{1}, \ldots q_{n}\right)= & \sum_{i=1}^{n} c\left(p_{0} ; q_{i}\right) f_{n-1}\left(p_{0}+q_{i} ; q_{1}, \ldots, \check{q}_{i}, \ldots, q_{n}\right) \\
& +\int_{\mathbb{R}^{d}} \overline{c\left(p_{0} ; q\right)} f_{n+1}\left(p_{0}-q ; q_{1}, \ldots q_{n}, q\right) d q
\end{aligned}
$$

with the convention that a sum over a void set is 0 , and where the notation $\check{q}$ means that the variable $q$ is omitted. The properties of the functions $\varepsilon$ and $c$ will be given in detail later. Notice that, with the minimal assumptions: $\varepsilon$ is a positive real function and the function $c$ is bounded and with sufficiently rapid decay for $q \rightarrow \infty$, the operator $H$ is self-adjoint and bounded from below.

A first simplification in the spectral analysis of $H$ comes from the conservation of the total momentum, i.e. from the fact that $H$ commutes with the operator

$$
(\hat{P} F)_{n}\left(p_{0} ; q_{1}, \ldots q_{n}\right)=\left(p_{0}+\sum_{i=1}^{n} q_{i}\right) f_{n}\left(p_{0} ; q_{1}, \ldots, q_{n}\right), n \geq 0 .
$$

As a consequence, $\mathcal{H}$ can be written as a direct integral of Hilbert spaces $\mathcal{H}(p)$

$$
\mathcal{H}=\int_{\mathbb{R}^{d}}^{\oplus} \mathcal{H}(p) d p
$$

which reduces both $\hat{P}$ and $H$, i.e. induces the decompositions

$$
\hat{P}=\int_{\mathbb{R}^{d}}^{\oplus} p I d p, \quad H=\int_{\mathbb{R}^{d}}^{\oplus} H_{p} d p
$$

where $I$ (the unit operator) and $H_{p}$ are operators in $\mathcal{H}(p)$. For a vector $F$ as given in (1.1), we get the representation:

$$
F=\int_{\mathbf{R}^{d}}^{\oplus} \hat{F}_{p} d p
$$

where $\hat{F}_{p}=\left\{\hat{f}_{p, n}\right\}_{n \geq 0}$ and $\hat{f}_{p, n}$ is the restriction of $f_{n}$ to the hyperplane $p_{0}+\sum_{i=1}^{n} q_{i}=p$. The spaces $\mathcal{H}(p)$ will be identified with $\mathcal{F}=\mathcal{F}_{\text {sym }}\left(L^{2}\left(\mathbb{R}^{d}\right)\right)$ by means of the unitaries

$$
\left(U_{p} \hat{F}_{p}\right)_{n}\left(q_{1}, \ldots q_{n}\right)=f_{n}\left(p-\sum_{i=1}^{n} q_{i} ; q_{1}, \ldots, q_{n}\right) .
$$


With this identification, the action of $H_{p}$ in $\mathcal{F}$ is given by the formula

$$
\begin{aligned}
& \left(H_{p} F\right)_{n}\left(q_{1}, \ldots q_{n}\right)=e_{n, p}^{0}\left(q_{1}, \ldots, q_{n}\right) f_{n}\left(q_{1}, \ldots, q_{n}\right) \\
& +\alpha \sum_{i=1}^{n} c\left(p-\sum_{j=1}^{n} q_{j} ; q_{i}\right) f_{n-1}\left(q_{1}, \ldots, \check{q}_{i}, \ldots, q_{n}\right) \\
& +\alpha \int_{\mathbb{R}^{d}} c\left(p-q-\sum_{j=1}^{n} q_{j} ; q\right) f_{n+1}\left(q_{1}, \ldots q_{n}, q\right) d q,
\end{aligned}
$$

where

$$
e_{n, p}^{0}\left(q_{1}, \ldots, q_{n}\right)=\frac{1}{2}\left(p-\sum_{i=1}^{n} q_{i}\right)^{2}+\sum_{i=1}^{n} \varepsilon\left(q_{i}\right),
$$

The functions $\varepsilon$ and $c$ are supposed to fulfill the following conditions:

1. $\varepsilon(q)$ is a convex, non-decreasing function of $|q|$ and there exists $c_{o}>0$, such that

$$
\varepsilon\left(q_{1}\right)+\varepsilon\left(q_{2}\right) \geq \varepsilon\left(q_{1}+q_{2}\right)+c_{o}, \forall q_{1}, q_{2} \in \mathbb{R}^{d} .
$$

Also, we need stronger regularity properties: $\varepsilon \in C^{\infty}\left(\mathbb{R}^{d}\right)$ and it has bounded derivatives, i.e. there exists $R>0$, such that for all multi-indices $\alpha=\left(\alpha_{1}, \ldots, \alpha_{d}\right) \neq$ 0 ,

$$
\sup _{q \in \mathbb{R}^{d}}\left|\partial_{q}^{\alpha} \varepsilon(q)\right| \leq R
$$

where

$$
\partial_{q}^{\alpha}=\frac{\partial^{|\alpha|}}{\partial q_{1}^{\alpha_{1}} \ldots \partial q_{d}^{\alpha_{d}}}, q=\left(q_{1}, \ldots, q_{d}\right), \quad|\alpha|=\sum_{i=1}^{d} \alpha_{i} .
$$

The following are physically interesting examples of such functions:

$$
\begin{aligned}
& \text { a) } \varepsilon(q)=\varepsilon(0)>0 \\
& \text { b) } \varepsilon(q)=\sqrt{q^{2}+m^{2}}+c_{o}, \quad m>0, c_{o}>0
\end{aligned}
$$

2. $c(p, q)$ is sufficiently smooth and there exists a bounded, rapidly decreasing function $h: \mathbb{R}^{d} \rightarrow \mathbb{R}_{+}$dominating $c$ and all its derivatives, i.e., for all multi-indices $\alpha, \beta$ there exists $C_{\alpha, \beta}>0\left(C_{0,0}=1\right)$, such that

$$
\left|\partial_{p}^{\alpha} \partial_{q}^{\beta} c(p ; q)\right| \leq C_{\alpha, \beta} h(q), \forall p, q \in \mathbb{R}^{d} .
$$

We are concerned here with the study by perturbation theory of the (lower part of the) spectrum of the Hamiltonian (1.9) for every fixed $p$ :

$$
H_{p}=H_{p}^{(0)}+\alpha H_{p, i n t}
$$

where $H_{p}^{(0)}$ denotes the first term, and $\alpha H_{p, \text { int }}$ the other terms, of equation(1.9); sometimes, for notational simplicity, the index $p$ will be omitted. 
The spectrum of $H_{p}^{(0)}$ consists of the eigenvalue $\frac{1}{2} p^{2}$, corresponding to the bare particle, and branches of continuous spectrum $e_{n, p}^{0}\left(q_{1}, \ldots, q_{n}\right)$, corresponding to bare particle $+n$ boson states, starting at the thresholds

$$
\lambda_{n}^{0}(p)=\min _{q_{1}, \ldots, q_{n}} e_{n, p}^{0}\left(q_{1}, \ldots, q_{n}\right) .
$$

Remark that, in view of the convexity of $p^{2}$ and $\varepsilon$, the minimum of $e_{1, p}^{0}\left(q_{1}\right)$ is attained at a single point $q_{1}^{0}$, which is its unique critical point and is nondegenerate. Moreover, as a consequence of the inequality (1.11),

$$
\lambda_{n}^{0}(p) \geq \lambda_{n-1}^{0}(p)+c_{o}
$$

The main result of the paper is summarized in the following:

\section{Theorem 1.1}

1) For any $d \geq 3$, there exists $\alpha_{0}=\alpha_{0}(d)$ such that, for any $\alpha<\alpha_{0}$ there exist functions $\lambda_{1}(p)<\lambda_{2}(p)$, with $\lambda_{1}(p)<\lambda_{1}^{0}(p), \lambda_{2}(p)<\lambda_{2}^{0}(p)$, and a bounded domain $G^{(0)} \subset \mathbb{R}^{d}$, such that the spectrum of $H_{p}$ in $\left(-\infty, \lambda_{1}(p)\right)$ consists of one nondegenerate eigenvalue $\xi_{p}^{(0)}$ if $p \in G^{(0)}$ and is void if $p \notin G^{(0)}$. Moreover, $\xi_{p}^{(0)}<p^{2} / 2$ and $\lim _{p^{\prime} \rightarrow p \in \partial G^{(0)}} \xi_{p^{\prime}}^{(0)}=\lambda_{1}(p)$ , where $\partial G^{(0)}$ denotes the boundary of the domain $G^{(0)}$. The associated eigenvector $F_{p}^{(0)}$ is the ground state of $H_{p}$.

2) For any $\kappa \in\left(\lambda_{1}(p), \lambda_{2}(p)\right)$ and any $p \in \mathbb{R}^{d}$, there exists $\bar{\alpha}_{0}=\bar{\alpha}_{0}(\kappa, p, d)$, such that for any $\alpha<\bar{\alpha}_{0}$ there exists a domain $G_{p}^{(1), \kappa} \subset \mathbb{R}^{d}$, a $C^{\infty}$-function $\xi_{p}^{\kappa}: G_{p}^{(1), \kappa} \rightarrow\left[\lambda_{1}(p), \kappa\right]$ and a subspace $\mathcal{H}_{1}^{\kappa}(p) \subset \mathcal{F}$ invariant for $H_{p}$, such that the restriction of $H_{p}$ to $\mathcal{H}_{1}^{\kappa}(p)$ is unitarily equivalent to the operator of multiplication by the function $\xi_{p}^{\kappa}$ in $L^{2}\left(G_{p}^{(1), \kappa}, d q\right)$ . Thereby, for $\kappa_{1}<\kappa_{2} \in\left(\lambda_{1}(p), \lambda_{2}(p)\right)$ one gets $G_{p}^{(1), \kappa_{1}} \subset G_{p}^{(1), \kappa_{2}}$ and $\xi_{p}^{\kappa_{1}}=\left.\xi_{p}^{\kappa_{2}}\right|_{G_{p}^{(1), \kappa_{1}}}$.

Remark 1.2 Refining slightly the technique of this paper, one can reach $\kappa=\lambda_{2}(p)$ if the dimension $d$ is sufficiently large, i.e. $\bar{\alpha}_{0}(\cdot, p, d)$ is bounded away from zero, and the whole one-boson subspace $\mathcal{H}_{1}^{\kappa=\lambda_{2}(p)}(p)$ and the function $\xi_{p}^{\kappa=\lambda_{2}(p)}$ can be constructed.

\section{Outline of the proof}

We shall present first the strategy we adopt in proving Theorem 1.1, in order not to obscure it by the cumbersome calculations to be done.

Our constructions involve the resolvent of $H_{p}$ :

$$
R(z)=\left(H_{p}-z I\right)^{-1} .
$$

We have therefore to solve, for any $L \in \mathcal{F}$, the equation:

$$
\left(H_{p}-z I\right) F=L \text {. }
$$


We split the space $\mathcal{F}$ as an orthogonal sum $\mathcal{H}^{(\leq 1)} \oplus \mathcal{H}^{(\geq 2)}$, corresponding to number $n$ of bare Bosons $\leq 1$, and $\geq 2$, respectively, and denote $\Pi_{1}, \Pi_{2}$ the corresponding orthogonal projections. Hence, $F=F_{1}+F_{2}$, where $F_{1}=\Pi_{1} F=\left\{f_{0}, f_{1}, 0,0, \ldots\right\}, F_{2}=\Pi_{2} F=$ $\left\{0,0, f_{2}, \ldots\right\}$ and similarly the vector $L=L_{1}+L_{2}$. Then the operator $H_{p}$ has a matrix representation:

$$
H_{p}=\left(\begin{array}{cc}
A_{11} & \alpha A_{12} \\
\alpha A_{21} & A_{22}
\end{array}\right)
$$

where $A_{i i}=\Pi_{i} H_{p} \Pi_{i}, \alpha A_{i j}=\Pi_{i} H_{p} \Pi_{j}(i \neq j)$, in terms of which equation (2.2) writes as

$$
\left\{\begin{array}{l}
\left(A_{11}-z I\right) F_{1}+\alpha A_{12} F_{2}=L_{1} \\
\alpha A_{21} F_{1}+\left(A_{22}-z I\right) F_{2}=L_{2}
\end{array} .\right.
$$

We define

$$
\lambda_{2}(p)=\inf \operatorname{spec}\left(A_{22}\right)
$$

By the variational principle,

$$
\lambda_{2}(p)=\inf _{F \in \mathcal{F} ;\|F\|=1}\left(\Pi_{2} F, H_{p} \Pi_{2} F\right) \leq \lambda_{2}^{0}(p)
$$

as $\lambda_{2}^{0}(p)$ is obtained as the infimum of the same expression taken over the subspace $\mathcal{H}^{(\leq 2)}$ of vectors $F$ with at most two bare bosons. Likewise, we define

$$
\lambda_{1}(p)=\inf _{F \in \mathcal{H}^{(\geq 1)} ;\|F\|=1}\left(F, H_{p} F\right) \leq \lambda_{1}^{0}(p)
$$

the infimum of the spectrum of the restriction of $H_{p}$ to the subspace $\mathcal{H}^{(\geq 1)}$ with at least one bare boson.

For $z$ in the resolvent set of $A_{22}$, the second equation in (2.4) can be solved for $F_{2}$

$$
F_{2}=\left(A_{22}-z I\right)^{-1}\left(L_{2}-\alpha A_{21} F_{1}\right),
$$

and hence one arrives at the following equation for $F_{1}$

$$
\left(A_{11}-\alpha^{2} A_{12}\left(A_{22}-z I\right)^{-1} A_{21}-z I\right) F_{1}=L_{1}-\alpha A_{12}\left(A_{22}-z I\right)^{-1} L_{2} .
$$

Let now restrict to real $z=\xi$ and consider the family of self-adjoint operators acting in $\mathcal{H}^{(\leq 1)}$ :

$$
A_{p}(\xi)=A_{11}-\alpha^{2} A_{12}\left(A_{22}-\xi I\right)^{-1} A_{21}, \xi \in\left(-\infty, \lambda_{2}(p)\right) .
$$

We shall show that, under our assumptions and for $\xi \leq \kappa \in\left(\lambda_{1}(p), \lambda_{2}(p)\right), A_{p}(\xi)$ are generalized Friedrichs operators, i.e. each operator $A_{p}(\xi)=A$ allows in the space $\mathcal{H}^{(\leq 1)}=\mathbb{C} \oplus L^{2}\left(\mathbb{R}^{d}, d q\right)$ a representation of the form:

$$
\begin{aligned}
& (A F)_{0}=e^{(0)} f_{0}+\alpha \int \bar{v}(q) f_{1}(q) d q \\
& (A F)_{1}=\alpha v(q) f_{0}+a(q) f_{1}(q)+\alpha^{2} \int D\left(q, q^{\prime}\right) f_{1}\left(q^{\prime}\right) d q^{\prime},
\end{aligned}
$$

where $F=\left(f_{0}, f_{1}\right) \in \mathcal{H}^{(\leq 1)}$. Here $v(q), a(q)$ and the kernel $D\left(q, q^{\prime}\right)$ fulfill a set of smoothness conditions (given in detail in Section 4.1), $a(q)$ is bounded from below and 
grows at most linearly at $\infty$, and $v(q), D\left(q, q^{\prime}\right)$ decrease fast at $\infty$. Such operators allow, for small $\alpha$, a complete spectral analysis (see [6]-8] and Section 4.1] below), namely, letting aside the possible eigenvalue $e_{p}$ (ground state), they are unitarily equivalent to the operator of multiplication by $a(q)$ in $L_{2}\left(\mathbb{R}^{d}, d q\right)$.

Let us denote, for given $p$ and $\xi$, by $a_{p}(\xi, q)$ the function $a(q)$ entering equation (2.11) written for $A_{p}(\xi)$. In essence, the key to the spectral analysis of $H_{p}$ lies the following remark:

Remark 2.1 Let $F=F_{1}+F_{2} \quad\left(F_{1} \in \mathcal{H}^{(\leq 1)}, F_{2} \in \mathcal{H}^{(\geq 2)}\right)$ be (generalized) eigenvector of $H_{p}$ with eigenvalue $\xi$. Then, by equation (2.9), $F_{1}$ is a (generalized) eigenvector of the operator $A_{p}(\xi)$ with the same eigenvalue $\xi$. Conversely, suppose that $F_{\xi, 1}$ is the eigenvector of $A_{p}(\xi)$ of eigenvalue $e_{p}(\xi)$ (whenever it exists). If the equation

$$
e_{p}(\xi)=\xi
$$

has a solution $\xi_{p}^{(0)}$, then $F=F_{\xi_{p}^{(0)}, 1}+F_{\xi_{p}^{(0)}, 2}$, where

$$
F_{\xi_{p}^{(0)}, 2}=-\alpha\left(A_{22}-\xi_{p}^{(0)} I\right)^{-1} A_{21} F_{\xi_{p}^{(0)}, 1}
$$

is an eigenvector of $H_{p}$ with eigenvalue $\xi_{p}^{(0)}$. Likewise, let for a given $p, F_{\xi, 1}^{q}$ be a generalized eigenvector of the operator $A_{p}(\xi)$ corresponding to the eigenvalue $a_{p}(\xi, q)$ and $\xi_{p}(q)$ be a solution of the equation

$$
a_{p}(\xi, q)=\xi
$$

Then for $F_{1}^{q}=F_{\xi(q), 1}^{q}$ and

$$
F_{2}^{q}=-\alpha\left(A_{22}-\xi(q) I\right)^{-1} A_{21} F_{1}^{q}
$$

the vector $F^{q}=F_{1}^{q}+F_{2}^{q}$ is a generalized eigenvector of $H_{p}$ for the eigenvalue $\xi_{p}(q)$.

The domain $G^{(0)}$ is identified with the set of $p$ for which equation (2.12) has a solution. For any given $p, G_{p}^{(1), \kappa}$ is the set of $q$ for which equation (2.13) has a solution $\xi(q) \leq \kappa$. The constructions of the subspace $\mathcal{H}_{1}^{\kappa}(p)$ and of the unitary equivalence of the operator $\left.H_{p}\right|_{\mathcal{H}_{1}^{\kappa}(p)}$ to the multiplication by $\xi_{p}^{\kappa}(q)=\xi_{p}(q)$ are done in the standard way in terms of the family $\left\{F^{q}\right\}_{q \in G_{p}^{(1), \kappa}}$ of generalized eigenvectors of $H_{p}$.

\section{Elimination of the many-body components}

In this section we study perturbatively the solution (2.8) and derive its main properties of interest to us. By virtue of equation (1.14) and the inequality (2.6), one can factorize the unperturbed (diagonal) part $H_{p}^{(0)}-z$ for $z \notin\left[\lambda_{2}(p), \infty\right)$, and bring the second equation (2.4) to the form of the equivalent fixed-point equation:

$$
F_{2}+Q(z) F_{2}=\alpha\left(H_{p}^{(0)}-z\right)^{-1} L_{2}-G F_{1},
$$


where

$$
\begin{gathered}
Q(z)=\alpha\left(H_{p}^{(0)}-z\right)^{-1} \Pi_{2} H_{p, i n t} \Pi_{2}, \\
G=\alpha\left(H_{p}^{(0)}-z\right)^{-1} \Pi_{2} H_{p, i n t} \Pi_{1},
\end{gathered}
$$

Explicitly, the vector $G F_{1}$ has the form $\left\{0,0, g_{2}, 0, \ldots\right\}$ with

$$
g_{2}\left(q_{1}, q_{2}\right)=\frac{\alpha\left[c\left(p-q_{1}-q_{2} ; q_{1}\right) f_{1}\left(q_{2}\right)+c\left(p-q_{1}-q_{2} ; q_{2}\right) f_{1}\left(q_{1}\right)\right]}{e_{2, p}^{0}\left(q_{1}, q_{2}\right)-z} .
$$

Lemma 3.1 For every $\kappa \in\left(\lambda_{1}(p), \lambda_{2}(p)\right)$ there exists $\alpha_{0}(\kappa)$ such that, for any $\alpha<\alpha_{0}(\kappa)$, and any $z \in \mathbb{C}$ with $\mathfrak{R e} z=\xi \leq \kappa,\|Q(z)\|<1 / 2$, therefore equation (3.1) has a unique solution $F_{2}$ for every $f_{1} \in L^{2}\left(\mathbb{R}^{d}, d q\right)$ and $L_{2} \in \mathcal{H}^{(\geq 2)}$.

Proof : We write $Q(z)$ as a sum of its creation and annihilation parts: $Q(z)=Q^{\prime}+Q^{\prime \prime}$, with

$$
\begin{aligned}
& \left(Q^{\prime} F\right)_{n}\left(q_{1}, \ldots, q_{n}\right) \\
& = \begin{cases}0, & n=2 \\
\alpha\left(e_{n, p}^{0}\left(q_{1}, \ldots, q_{n}\right)-z\right)^{-1} \sum_{i=1}^{n} c\left(p-\sum_{j} q_{j} ; q_{i}\right) f_{n-1}\left(\ldots \check{q}_{i} \ldots\right), & n>2\end{cases} \\
& \left(Q^{\prime \prime} F\right)_{n}\left(q_{1}, \ldots, q_{n}\right) \\
& =\alpha\left(e_{n, p}^{0}\left(q_{1}, \ldots, q_{n}\right)-z\right)^{-1} \int \overline{c\left(p-\sum_{j} q_{j}-q ; q\right)} f_{n+1}\left(q_{1}, \ldots, q_{n}, q\right) d q \text {, } \\
& n \geq 2
\end{aligned}
$$

By condition (1.11) and the inequality (2.6), one gets for $\xi \leq \kappa$

$$
\left|e_{n, p}^{0}\left(q_{1}, \ldots, q_{n}\right)-z\right| \geq e_{n, p}^{0}\left(q_{1}, \ldots, q_{n}\right)-\xi \geq(n-2) c_{o}+\lambda_{2}(p)-\kappa,
$$

therefore, by virtue of (1.13),

$$
\left\|\left(Q^{\prime} F\right)_{n}\right\|_{L^{2}\left(\mathbb{R}^{d n}\right)} \leq \frac{n \alpha \cdot\|h\|_{L^{2}\left(\mathbb{R}^{d}\right)} \cdot\left\|f_{n-1}\right\|_{L^{2}\left(\mathbf{R}^{d(n-1)}\right)}}{(n-2) c_{o}+\lambda_{2}(p)-\kappa}, n>2,
$$

while, for $n=2$, the norm vanishes. Therefore,

$$
\begin{aligned}
& \left\|Q^{\prime} F\right\|_{\mathcal{H}^{(\geq 2)}}^{2}=\sum_{n=2}^{\infty} \frac{1}{n !} \cdot\left\|\left(Q^{\prime} F\right)_{n}\right\|_{L^{2}\left(\mathbb{R}^{d n}\right)}^{2} \\
& \leq \alpha^{2}\|h\|_{L^{2}\left(\mathbb{R}^{d}\right)}^{2} \max _{n \geq 3}\left[n\left((n-2) c_{o}+\lambda_{2}(p)-\kappa\right)^{-2}\right] \\
& \times \sum_{n=3}^{\infty} \frac{1}{(n-1) !} \cdot\left\|f_{n-1}\right\|_{L^{2}\left(\mathbb{R}^{d(n-1)}\right)}^{2} \\
& \leq \alpha^{2}\|h\|_{L^{2}\left(\mathbb{R}^{d}\right)}^{2} 3\left(c_{o}+\lambda_{2}(p)-\kappa\right)^{-2}\|F\|_{\mathcal{H}(\geq 2)}^{2}
\end{aligned}
$$


implying that $\left\|Q^{\prime}\right\| \leq \alpha \cdot\|h\|_{L^{2}\left(\mathbb{R}^{d}\right)} \sqrt{3} /\left(c_{o}+\lambda_{2}(p)-\kappa\right)$. A similar calculation shows that $\left\|Q^{\prime \prime}\right\| \leq \alpha \cdot\|h\|_{L^{2}\left(\mathbb{R}^{d}\right)} \sqrt{3} /\left(\lambda_{2}(p)-\kappa\right)$. This finishes the proof of the lemma.

Let us denote by $F_{2}^{0}\left(z, f_{1}\right)=\left\{f_{n}^{0}(z ; \cdot) ; n \geq 2\right\}$ the solution of equation (3.1) for $L_{2}=$ 0 . Under the conditions of Lemma 3.1 and taking into account equation (3.4), we get that $\left\|F_{2}^{0}\left(z, f_{1}\right)\right\|_{\mathcal{H}(\geq 2)} \leq C \alpha\left\|f_{1}\right\|_{L^{2}\left(\mathbb{R}^{d}\right)}$. From now on, we shall denote by $S(z)$ the linear operator:

$$
\mathcal{H}^{(\leq 1)} \ni f_{1} \stackrel{S(z)}{\longmapsto} F_{2}^{0}\left(z, f_{1}\right) \in \mathcal{H}^{(\geq 2)} .
$$

To proceed further with the analysis we need more information about the structure and regularity of the solution $F_{2}^{0}\left(z, f_{1}\right)$. To this aim, we shall solve equation (3.1) with $L_{2}=0$. In particular, we shall show that the components of $F_{2}^{0}\left(z, f_{1}\right)$ have the representation

$$
\begin{array}{r}
f_{n}^{0}\left(z ; q_{1}, \ldots, q_{n}\right)= \\
\sum_{i=1}^{n} b_{n}\left(q_{1}, \ldots, \check{q}_{i} . ., q_{n} ; q_{i}\right) f_{1}\left(q_{i}\right) \\
+\int d_{n}\left(q_{1}, \ldots, q_{n} ; q\right) f_{1}(q) d q
\end{array}
$$

where the functions $b_{n}\left(q_{1}, \ldots, q_{n-1} ; q_{n}\right)$ are symmetric in $q_{1}, \ldots, q_{n-1}$ and the functions $d_{n}\left(q_{1}, \ldots, q_{n} ; q\right)$ are symmetric in $q_{1}, \ldots, q_{n}, n \geq 2$. The functions $b_{n}$ and $d_{n}$ will be called coefficient functions.

A simple calculation shows that, if $F \in \mathcal{H}^{(\geq 2)}$ has the representation (3.9), then also $\hat{F}=Q(z) F$ has the same kind of representation, with coefficient functions

$$
\begin{aligned}
& \hat{b}_{n}\left(q_{1}, \ldots, q_{n-1} ; q_{n}\right)=\alpha\left(e_{n, p}^{0}\left(q_{1}, \ldots, q_{n}\right)-z\right)^{-1} \\
& \times\left[\sum_{i=1}^{n-1} c\left(p-\sum_{j=1}^{n} q_{j} ; q_{i}\right) b_{n-1}\left(q_{1}, \ldots, \check{q}_{i} . ., q_{n-1} ; q_{n}\right)\right. \\
& \left.+\int \bar{c}\left(p-\sum_{j=1}^{n} q_{j}-q ; q\right) b_{n+1}\left(q_{1}, \ldots, q_{n-1}, q ; q_{n}\right) d q\right] \\
& \hat{d}_{n}\left(q_{1}, \ldots, q_{n} ; q\right)=\alpha\left(e_{n, p}^{0}\left(q_{1}, \ldots, q_{n}\right)-z\right)^{-1} \\
& \times\left[\sum_{i=1}^{n} c\left(p-\sum_{j=1}^{n} q_{j} ; q_{i}\right) d_{n-1}\left(q_{1}, \ldots, \check{q}_{i} . ., q_{n} ; q\right)\right. \\
& +\int \bar{c}\left(p-\sum_{j=1}^{n} q_{j}-q^{\prime} ; q^{\prime}\right) d_{n+1}\left(q_{1}, \ldots, q_{n}, q^{\prime} ; q\right) d q^{\prime} \\
& \left.+\bar{c}\left(p-\sum_{j=1}^{n} q_{j}-q ; q\right) b_{n+1}\left(q_{1}, \ldots, q_{n} ; q\right)\right]
\end{aligned}
$$

Let now define the space $\mathcal{M}$ of all pairs $\mu=\left\{\left(b_{n}\right)_{n \geq 2},\left(d_{n}\right)_{n \geq 2}\right\}$ of sequences of bounded continuous functions, $b_{n}:\left(\mathbb{R}^{d}\right)^{(n-1)} \times \mathbb{R}^{d} \rightarrow \mathbb{C}, d_{n}:\left(\mathbb{R}^{d}\right)^{n} \times \mathbb{R}^{d} \rightarrow \mathbb{C}$, symmetric with respect to the first group of variables. Let them fulfill the following condition: there exists 
a constant $M$, such that,

$$
\begin{aligned}
& \sup _{q}\left|b_{n}\left(q_{1}, \ldots, q_{n-1} ; q\right)\right| \leq M \prod_{i=1}^{n-1} h\left(q_{i}\right), \\
& \left|d_{n}\left(q_{1}, \ldots, q_{n} ; q\right)\right| \leq M h(q) \prod_{i=1}^{n} h\left(q_{i}\right), \quad \forall n \geq 2
\end{aligned}
$$

where $h$ is the function appearing in equation (1.13). $\mathcal{M}$ is a Banach space with the norm

$$
\|\mu\|=\inf M
$$

where the infimum is taken over all $M$ for which the condition (3.12) holds. Clearly, equation (3.9) defines a continuous application of $\mathcal{H}^{(1)}$ into $\mathcal{H}^{(\geq 2)}$.

The linear operator $\Gamma(z)$ acting in $\mathcal{M}$ according to $\Gamma(z) \mu=\hat{\mu}$, where $\mu=\left\{\left(b_{n}\right)_{n \geq 2},\left(d_{n}\right)_{n \geq 2}\right\}$ and $\hat{\mu}=\left\{\left(\hat{b}_{n}\right)_{n \geq 2},\left(\hat{d}_{n}\right)_{n \geq 2}\right\}$ are related by (3.10), (3.11), translates in $\mathcal{M}$ the action of $Q(z)$. Then equation (3.1) with $L_{2}=0$ is transformed into

$$
\mu+\Gamma(z) \mu=\mu_{0}
$$

where $\mu_{0}=\left\{\left(b_{n}^{0}\right),\left(d_{n}^{0}\right)\right\}$ with $b_{n}^{0}=0, \forall n \geq 3, d_{n}^{0}=0, \forall n \geq 2$, and

$$
b_{2}^{0}\left(q_{1} ; q\right)=-\frac{\alpha c\left(p-q_{1}-q ; q_{1}\right)}{e_{2, p}^{0}\left(q_{1}, q\right)-z} .
$$

Lemma 3.2 For every $\kappa \in\left(\lambda_{1}(p), \lambda_{2}(p)\right)$ there exists $\tilde{\alpha}_{0}(\kappa)$ such that, for any $\alpha<\tilde{\alpha}_{0}(\kappa)$, and any $z \in \mathbb{C}$ with $\mathfrak{R e} z=\xi \leq \kappa,\|\Gamma(z)\|<1 / 2$, and $\mu_{0} \in \mathcal{M},\left\|\mu_{0}\right\| \leq \alpha /\left(\lambda_{2}(p)-\kappa\right)$. Therefore, equation 3.14) has a unique solution $\mu(z) \in \mathcal{M}$, which is an analytic function of $z$ in the half-plane $\mathfrak{R e} z \leq \kappa$. Moreover, for any $r \geq 1$, there exists $\tilde{\alpha}_{r}(\kappa)$, such that, for $\alpha<\tilde{\alpha}_{r}(\kappa)$, the components of $\mu(z)$ are $C^{r}$-functions of their arguments and the derivatives up to order $r$ satisfy estimates like (1.13), more precisely, for any multi-indices $\mathcal{A}_{n}=\left\{\alpha_{1}, \ldots, \alpha_{n}, \beta\right\}$ with $\left|\mathcal{A}_{n}\right|=\sum_{i=1}^{n}\left|\alpha_{i}\right|+\beta \leq r$, where $\alpha_{i}=\left(\alpha_{i}^{1}, \ldots, \alpha_{i}^{d}\right), \beta=\left(\beta^{1}, \ldots, \beta^{d}\right)$, there exist constants $C\left(\mathcal{A}_{n}\right), \tilde{C}\left(\mathcal{A}_{n}\right)$, such that, for any $n \geq 2$ and for all $z$ in the halfplane the following inequalities hold:

$$
\begin{gathered}
\left|\partial^{\mathcal{A}_{n-1}} b_{n}\left(z ; q_{1}, \ldots, q_{n-1} ; q\right)\right| \leq \alpha\left(\lambda_{2}(p)-\kappa\right)^{-1} \cdot C\left(\mathcal{A}_{n-1}\right) \cdot \prod_{i=1}^{n-1} h\left(q_{i}\right) \\
\left|\partial^{\mathcal{A}_{n}} d_{n}\left(z ; q_{1}, \ldots, q_{n} ; q\right)\right| \leq \alpha\left(\lambda_{2}(p)-\kappa\right)^{-1} \cdot C\left(\mathcal{A}_{n}\right) \cdot h(q) \prod_{i=1}^{n} h\left(q_{i}\right) \\
\left|\frac{d}{d z} \partial^{\mathcal{A}_{n-1}} b_{n}\left(z ; q_{1}, \ldots, q_{n-1} ; q\right)\right| \leq \alpha\left(\lambda_{2}(p)-\kappa\right)^{-2} \cdot \tilde{C}\left(\mathcal{A}_{n-1}\right) \cdot \prod_{i=1}^{n-1} h\left(q_{i}\right) \\
\left|\frac{d}{d z} \partial^{\mathcal{A}_{n}} d_{n}\left(z ; q_{1}, \ldots, q_{n} ; q\right)\right| \leq \alpha\left(\lambda_{2}(p)-\kappa\right)^{-2} \cdot \tilde{C}\left(\mathcal{A}_{n}\right) \cdot h(q) \prod_{i=1}^{n} h\left(q_{i}\right)
\end{gathered}
$$


where $\partial^{\mathcal{A}_{n}}=\left(\prod_{i=1}^{n} \partial_{q_{i}}^{\alpha_{i}}\right) \partial_{q}^{\beta}$. The vector $F_{2}^{0}\left(z ; f_{1}\right)$ given by equation (3.9), having as coefficient functions the components $b_{n}, d_{n}$ of $\mu(z)$, belongs to $\mathcal{H}^{(\geq 2)}$ and is the unique solution of equation (3.1) for $L_{2}=0$.

Proof: Suppose $\mu \in \mathcal{M},\|\mu\|=1$, i.e. $\left(b_{n}\right)_{n \geq 2},\left(d_{n}\right)_{n \geq 2}$ satisfy the estimates (3.12) with $M=1$. Then, $\Gamma(z) \mu=\hat{\mu}$ of components (3.10), (3.11) satisfies the same estimates with

$$
\hat{A}=\alpha \cdot \max _{n \geq 2} \frac{n+1+\|h\|^{2}}{(n-2) c_{o}+\lambda_{2}(p)-\kappa}=\frac{\alpha\left(3+\|h\|^{2}\right)}{\lambda_{2}(p)-\kappa} .
$$

Therefore, $\|\Gamma(z)\|<1 / 2$ for $\alpha$ sufficiently small. The estimate of $\left\|\mu_{0}\right\|$ is immediate, therefore $\|\mu(z)\| \leq 2 \alpha\left(\lambda_{2}(p)-\kappa\right)^{-1}$.

So, we are left with the proof of the smoothness of the coefficient functions, equations (3.16), (3.17). We shall consider only the first derivatives, i.e. $\left|\mathcal{A}_{n}\right|=1$. Consider the subspace $\mathcal{M}_{1} \subset \mathcal{M}$ of all $\mu$ with differentiable components $\left(b_{n}\right)_{n \geq 2},\left(d_{n}\right)_{n \geq 2}$ for which there exists $M_{1}>0$, such that

$$
\begin{aligned}
& \max \left\{\max _{1 \leq i \leq n-1}\left|\nabla_{q_{i}} b_{n}\left(q_{1}, \ldots, q_{n-1} ; q\right)\right|,\left|\nabla_{q} b_{n}\left(q_{1}, \ldots, q_{n-1} ; q\right)\right|\right\} \leq M_{1} \prod_{i=1}^{n-1} h\left(q_{i}\right), \\
& \max \left\{\max _{1 \leq i \leq n}\left|\nabla_{q_{i}} d_{n}\left(q_{1}, \ldots, q_{n} ; q\right)\right|,\left|\nabla_{q} d_{n}\left(q_{1}, \ldots, q_{n} ; q\right)\right|\right\} \leq M_{1} h(q) \prod_{i=1}^{n} h\left(q_{i}\right),
\end{aligned}
$$

which is a Banach space with norm $\|\mu\|_{1}=\max \left\{\|\mu\|\right.$, inf $\left.M_{1}\right\}$, where inf is taken over all $M_{1}$ fulfilling (3.18). We show that, for small $\alpha, \Gamma(z)$ is a contraction in $\mathcal{M}_{1}$, as well. Taking derivatives with respect, say, to $q_{1}$ in equation (3.10), one obtains

$$
\begin{aligned}
& \nabla_{q_{1}} \hat{b}_{n}\left(q_{1}, \ldots, q_{n-1} ; q_{n}\right) \\
= & -\alpha\left(e_{n, p}^{0}\left(q_{1}, \ldots, q_{n}\right)-z\right)^{-2} \nabla_{q_{1}} e_{n, p}^{0}\left(q_{1}, \ldots, q_{n-1}, q_{n}\right) \\
& \times\left[\sum_{i=1}^{n-1} c\left(p-\sum_{j=1}^{n} q_{j} ; q_{i}\right) b_{n-1}\left(\ldots, \check{q}_{i}, \ldots ; q_{n}\right)\right. \\
& \left.+\int \bar{c}\left(p-\sum_{j=1}^{n} q_{j}-q ; q\right) b_{n+1}\left(q_{1}, \ldots, q_{n-1}, q ; q_{n}\right) d q\right] \\
+\alpha\left(e_{n, p}^{0}\left(q_{1}, \ldots, q_{n}\right)-z\right)-1 & {\left[\left(\nabla_{q} c\left(p-\sum_{j=1}^{n} q_{j} ; q_{1}\right)-\sum_{i=1}^{n-1} \nabla_{p} c\left(p-\sum_{j=1}^{n} q_{j} ; q_{i}\right)\right) b_{n-1}\left(\ldots \check{q}_{i} \ldots ; q_{n}\right)\right.} \\
& -\int \nabla_{p} \bar{c}\left(p-\sum_{j=1}^{n} q_{j}-q ; q\right) b_{n+1}\left(q_{1}, \ldots, q_{n-1}, q ; q_{n}\right) d q \\
+ & \sum_{i=2}^{n-1} c\left(p-\sum_{j=1}^{n} q_{j} ; q_{i}\right) \nabla_{q_{1}} b_{n-1}\left(q_{1}, \ldots, \check{q}_{i} . ., q_{n-1} ; q_{n}\right) \\
+ & \left.\int \bar{c}\left(p-\sum_{j=1}^{n} q_{j}-q ; q\right) \nabla_{q_{1}} b_{n+1}\left(q_{1}, \ldots, q_{n-1}, q ; q_{n}\right) d q\right] .
\end{aligned}
$$


Here, $\nabla_{p} c$ and $\nabla_{q} c$ denote the gradient of the function $c(p, q)$ with respect to the first, respectively the second, argument. Similar expressions are obtained for $\nabla_{q_{i}} \hat{d}_{n}, \nabla_{q_{n}} \hat{b}_{n}$, and $\nabla_{q} \hat{d}_{n}$. Suppose that $\|\mu\|_{1}=1$. Then, using the simple estimate

$$
\left|\frac{\nabla_{q_{1}} e_{n, p}^{0}\left(q_{1}, \ldots, q_{n-1}, q_{n}\right)}{e_{n, p}^{0}\left(q_{1}, \ldots, q_{n}\right)-z}\right| \leq \bar{R}
$$

where $\bar{R}$ is a constant independent of $n$, and also the assumption (1.13), one obtains

$$
\|\hat{\mu}\|_{1} \leq \frac{\alpha\left(a+\|h\|^{2}\right)(\bar{R}+\bar{C}+b)}{\lambda_{2}(p)-\kappa},
$$

where $a$ and $b$ are absolute constants and $\bar{C}=\max \left\{\left|\nabla_{p} c(p, q)\right|,\left|\nabla_{q} c(p, q)\right|\right\}$. Equation (3.21) shows that $\Gamma(z)$ leaves $\mathcal{M}_{1}$ invariant and that $\|\Gamma(z)\|<1 / 2$ for $\alpha$ sufficiently small. Since $\mu_{0} \in \mathcal{M}_{1}$, and

$$
\left\|\mu_{0}\right\|_{1}<2 \alpha\left(\lambda_{2}(p)-\kappa\right)^{-1} \max \{(\bar{R}+\bar{C}), 1\},
$$

we see that the solution $\mu(z)$ of equation (3.14) belongs to $\mathcal{M}_{1}$ and has norm of the order of $\alpha /\left(\lambda_{2}(p)-\kappa\right)$. This finishes the proof of the inequalities (3.16) in the case $r=1$. The higher values of $r$ can be treated similarly, with stronger limitations on $\alpha$.

Finally, $\mu(z)$ and its derivatives are analytic in the half-plane $\xi \leq \kappa^{\prime}$ for any $\kappa^{\prime} \in$ $\left(\kappa, \lambda_{2}(p)\right)$ and satisfy there inequalities like (3.16), implying (3.17) in $\xi \leq \kappa$. The lemma is proved.

Finally, going back to the system (2.4) with $L_{2}=0$, we remark that the solution $F_{2}^{0}\left(z ; f_{1}\right)$ of the second equation enters the first equation only through its first $(n=2)$ component, $f_{2}^{0}\left(z ; f_{1}\right)$, which, in view of equation (3.9) has the form:

$$
\begin{aligned}
f_{2}^{0}\left(z ; f_{1} ; q_{1}, q_{2}\right)=b_{2}\left(z ; q_{2} ; q_{1}\right) & f_{1}\left(q_{1}\right)+b_{2}\left(z ; q_{1} ; q_{2}\right) f_{1}\left(q_{2}\right) \\
& +\int d_{2}\left(z ; q_{1}, q_{2} ; q\right) f_{1}(q) d q .
\end{aligned}
$$

Inserting this representation into the first equation (2.4) and using the notations:

$$
\begin{aligned}
& m_{p}(z ; q)=\alpha \int \overline{c\left(p-q-q^{\prime} ; q^{\prime}\right)} b_{2}\left(z ; q^{\prime} ; q\right) d q^{\prime} \\
& D_{p}\left(z ; q, q^{\prime}\right)=\frac{1}{\alpha}\left[c\left(p-q-q^{\prime}, q^{\prime}\right) b_{2}\left(z ; q ; q^{\prime}\right)\right. \\
& \left.\quad+\int \overline{c\left(p-q^{\prime}-q^{\prime \prime}, q^{\prime \prime}\right)} d_{2}\left(z ; q, q^{\prime \prime} ; q^{\prime}\right) d q^{\prime \prime}\right]
\end{aligned}
$$

one arrives at the following system of equations for the $n=0,1$ components:

$$
\left\{\begin{array}{lll}
\left(e_{0, p}^{0}-z\right) f_{0} & +\alpha \int \overline{c(p-q, q)} f_{1}(q) d q & =l_{0} \\
\alpha c(p-q, q) f_{0} & +\left[a_{p}(z ; q)-z\right] f_{1}(q)+\alpha^{2} \int D_{p}\left(z ; q, q^{\prime}\right) f_{1}\left(q^{\prime}\right) d q^{\prime} & =l_{1}
\end{array}\right.
$$

where

$$
a_{p}(z ; q)=e_{1, p}^{0}(q)+m_{p}(z ; q)
$$


Corollary 3.3 For $z$ real, the function $a_{p}(z ; q)$ is real and the kernel $D_{p}\left(z ; q, q^{\prime}\right)$ is self-adjoint.

Indeed, the operator $V(z)$ defined by

$$
\left(V(z) f_{1}\right)(q)=m_{p}(z ; q) f_{1}(q)+\alpha^{2} \int D_{p}\left(z ; q, q^{\prime}\right) f_{1}\left(q^{\prime}\right) d q^{\prime}
$$

is equal to $-A_{12}\left(A_{22}-z I\right)^{-1} A_{21}$ appearing in equation (2.9), which is manifestly selfadjoint for real $z$.

Corollary 3.4 The following asymptotic formulae hold:

$$
m_{p}(z ; q)=-\alpha^{2} \int \frac{\left|c\left(p-q-q^{\prime} ; q^{\prime}\right)\right|^{2}}{e_{2, p}^{0}\left(q, q^{\prime}\right)-z} d q^{\prime}+O\left(\alpha^{3}\right),
$$

where $O\left(\alpha^{3}\right)$ is a $C^{1}$-function of norm $\left\|O\left(\alpha^{3}\right)\right\|_{1} \leq C \alpha^{3}$ for some constant $C$ depending on $\kappa$;

$$
D_{p}\left(z ; q, q^{\prime}\right)=-\frac{\overline{c\left(p-q-q^{\prime} ; q^{\prime}\right)} c\left(p-q-q^{\prime} ; q\right)}{e_{2, p}^{0}\left(q, q^{\prime}\right)-z}+O(\alpha),
$$

where $O(\alpha)$ is a smooth function bounded by $C \alpha h(q) h\left(q^{\prime}\right)$ for some constant $C$ depending on $\kappa$.

As a consequence of Lemma 3.2, the solution $\mu(z)$ has a series representation $\sum_{n=0}^{\infty}(-\Gamma(z))^{n} \mu_{0}$ convergent in $\mathcal{M}_{1}$, the $n$-th term of which is of the order $\alpha^{n}$, wherefrom the assertion.

\section{Study of the reduced system (3.25)}

\subsection{The generalized Friedrichs model (a digression)}

We collect here the needed information about the spectral representation of the generalized Friedrichs operator $A$ acting in $\mathcal{H}^{(\leq 1)}=\mathbb{C} \oplus L^{2}\left(\mathbb{R}^{d}, d q\right)$, equation (2.11). We shall study $A$ as a perturbation of $A_{0}=A(\alpha=0)$, so $\alpha>0$ is supposed sufficiently small to ensure the convergence.

In order to calculate the resolvent $R_{A}(z)$ of $A$, one has to solve

$$
\left\{\begin{aligned}
\left(e^{(0)}-z\right) f_{0}+\alpha \int \bar{v}(q) f_{1}(q) d q & =g_{0} \\
\alpha v(q) f_{0}+(a(q)-z) f_{1}(q)+\alpha^{2} \int D\left(q, q^{\prime}\right) f_{1}\left(q^{\prime}\right) d q^{\prime} & =g_{1}
\end{aligned}\right.
$$

for all $\left(g_{0}, g_{1}\right)=G \in \mathcal{H}^{(\leq 1)}$.

To this end the following assumptions are made: 
1. $a(q)$ is a real, sufficiently smooth function, and there exist constants $C_{1}, C_{2}, C_{3}$, such that

$$
\begin{gathered}
C_{1} \leq a(q) \leq C_{2}|q|^{2}+C_{3}, \\
|\nabla a(q)| \leq C_{2}(|q|+1), \\
\left|\partial^{\alpha} a(q)\right| \leq C_{2},|\alpha| \geq 2 ;
\end{gathered}
$$

$a(q)$ has a unique nondegenerate minimum $\bar{a}$ at $\bar{q}_{0}$ and no other critical points. We denote $I=[\bar{a}, \infty) \subset \mathbb{R}$ the range of the function $a$.

2. The function $v(q)$ is continuous and $|v(q)| \leq h(q)$, for some bounded, rapidly decreasing, positive $h$;

3. $a(q)$ restricted to the 0 -level of $v,\{q: v(q)=0\}$, is not constant;

4. The kernel $D\left(q, q^{\prime}\right)$ is sufficiently smooth and there exists a constant $N$, such that, for any multi-indices $\alpha, \beta$ with $|\alpha|,|\beta| \leq r=[d / 2]+2$

$$
\left|\partial_{q}^{\alpha} \partial_{q^{\prime}}^{\beta} D\left(q, q^{\prime}\right)\right| \leq N h(q) h\left(q^{\prime}\right)
$$

In solving equation (4.1) we proceed like outlined in Section 2 , i.e. we solve the second equation for $f_{1}$ in terms of $f_{0}$ and plug the solution in the first equation.

Let $B$ be the operator defined on its maximal domain in $L^{2}\left(\mathbb{R}^{d}, d q\right)$ by the formula:

$$
B f(q)=a(q) f(q)+\alpha^{2} \int D\left(q, q^{\prime}\right) f\left(q^{\prime}\right) d q^{\prime}
$$

We need its resolvent $R_{B}(z)=(B-z I)^{-1}$. We denote by $\mathcal{B}_{r}$ the Banach space of all kernels $D\left(q, q^{\prime}\right)$ satisfying condition 4 , i.e. which are $r$ times differentable and satisfy (4.3) for some $N$, endowed with the norm $\|D\|_{r}=\inf N$, where the infimum is taken over all $N$ for which (4.3) holds.

Lemma 4.1 For $\alpha$ sufficiently small and $z \notin I$ the resolvent $R_{B}(z)=(B-z I)^{-1}$ has the form

$$
\begin{aligned}
& \left(R_{B}(z) g\right)(q) \\
& =(a(q)-z)^{-1}\left[g(q)+\alpha^{2} \int K\left(\alpha, z ; q, q^{\prime}\right) g\left(q^{\prime}\right)\left(a\left(q^{\prime}\right)-z\right)^{-1} d q^{\prime}\right] \\
& g \in L^{2}\left(\mathbb{R}^{d}, d q\right)
\end{aligned}
$$

where the kernel $K(\alpha, z ; \cdot, \cdot) \in \mathcal{B}_{r}$ and its norm is bounded for $z \in \mathbb{C} \backslash I$. Moreover, $K$ is a $\mathcal{B}_{r}$-valued analytic function of $z$ on $\mathbb{C} \backslash I$ and its boundary values

$$
K^{ \pm}\left(\alpha, x ; q, q^{\prime}\right)=\lim _{\varepsilon \searrow 0} K\left(\alpha, x \pm i \varepsilon ; q, q^{\prime}\right)
$$

exist in $\mathcal{B}_{r}$ for all $x \in I$. Also, $K^{ \pm}(\alpha, x ; \cdot, \cdot)$ are $[(d-1) / 2]-1$ times differentiable as a $\mathcal{B}_{r}$-valued function of $x \in I$ and their last derivative with respect to $x$ is Hölder continuous of exponent $\gamma=1 / 3$ (actually of any $\gamma<1 / 2$ for even $d$ and any $\gamma<1$ for odd $d$ ). 
Remark 4.2 We shall express the last property of $K^{ \pm}$by saying that $I \ni x \longmapsto K^{ \pm}(\alpha, x ; \cdot, \cdot) \in$ $\mathcal{B}_{r}$ is $s+1 / 3$ times differentiable, where we put $s=[(d-1) / 2]-1$. In order to prove it, one has to find a constant $\tilde{N}$, such that for all multi-indices $\alpha, \beta$ with $|\alpha|,|\beta| \leq r=[d / 2]+2$ and $k=0,1, \ldots, s$ :

$$
\left|\partial_{x}^{k} \partial_{q}^{\alpha} \partial_{q^{\prime}}^{\beta} K^{ \pm}\left(\alpha, x ; q, q^{\prime}\right)\right| \leq \tilde{N} h(q) h\left(q^{\prime}\right)
$$

and

$$
\max _{x, y \in I ;|x-y| \leq 1} \frac{\left|\partial_{x}^{s} \partial_{q}^{\alpha} \partial_{q^{\prime}}^{\beta} K^{ \pm}\left(\alpha, x ; q, q^{\prime}\right)-\partial_{x}^{s} \partial_{q}^{\alpha} \partial_{q^{\prime}}^{\beta} K^{ \pm}\left(\alpha, y ; q, q^{\prime}\right)\right|}{|x-y|^{1 / 3}} \leq \tilde{N} h(q) h\left(q^{\prime}\right) .
$$

Proof of Lemma 4.1 Let $B_{0}=B(\alpha=0)$, i.e. the operator of multiplication with $a(q)$ and $D$ the integral operator of kernel $D\left(q, q^{\prime}\right)$. Then, denoting

$$
M=\alpha^{2} D\left(B_{0}-z\right)^{-1}
$$

which is an integral operator of kernel $\alpha^{2} D\left(q, q^{\prime}\right)\left(a\left(q^{\prime}\right)-z\right)^{-1}$, we have, formally, the expansion:

$$
\begin{array}{r}
R_{B}(z)=\left(B_{0}-z\right)^{-1}(I+M)^{-1}=\left(B_{0}-z\right)^{-1} \\
+\sum_{n=1}^{\infty}(-1)^{n}\left(B_{0}-z\right)^{-1} M^{n}
\end{array}
$$

where $\left(B_{0}-z\right)^{-1} M^{n}, n \geq 1$, are integral operators of kernels

$$
(a(q)-z)^{-1} L_{n}\left(\alpha, z ; q, q^{\prime}\right)\left(a\left(q^{\prime}\right)-z\right)^{-1}
$$

with

$$
L_{n}\left(\alpha, z ; q, q^{\prime}\right)=\alpha^{2 n} \int \ldots \int \frac{D\left(q, q_{1}\right) D\left(q_{1}, q_{2}\right) \ldots D\left(q_{n-1}, q^{\prime}\right)}{\prod_{i=1}^{n-1}\left(a\left(q_{i}\right)-z\right)} d q_{1} \ldots d q_{n-1} .
$$

We shall prove the convergence of the series (4.10) in $\mathcal{B}_{r}$ and, hence, show that $K$ satisfies all the assertions of the Lemma, by checking (by induction) the following properties of the function (4.11):

(i) $L_{n}(\alpha, z ; \cdot, \cdot) \in \mathcal{B}_{r}$ and

$$
\left\|L_{n}(\alpha, z ; \cdot, \cdot)\right\|_{r} \leq\left(C \alpha^{2}\right)^{n-1}
$$

where $C$ is a constant (to be specified later);

(ii) The limits

$$
\lim _{\varepsilon \searrow 0} L_{n}\left(\alpha, x \pm i \varepsilon ; q, q^{\prime}\right)=L_{n}^{ \pm}\left(\alpha, x ; q, q^{\prime}\right)
$$

exist in $\mathcal{B}_{r}$ for all $x \in I$;

(iii) $L_{n}^{ \pm}(\alpha, x ; \cdot, \cdot)$ are $s+1 / 3$ times differentiable, thereby they satisfy the estimates (4.7), (4.8) with $\tilde{N}=\left(C \alpha^{2}\right)^{n-1}$. 
Indeed, for $n=1$, i.e. for $D\left(q, q^{\prime}\right)$ these assertions hold obviously. For $\mathfrak{I m} z \geq 0$, we represent

$$
\begin{array}{r}
L_{n+1}\left(\alpha, z ; q, q^{\prime}\right)=\alpha^{2} \int D(q, \bar{q}) L_{n}\left(\alpha, z ; \bar{q}, q^{\prime}\right)(a(\bar{q})-z)^{-1} d \bar{q} \\
=i \alpha^{2} \int_{0}^{\infty} d t e^{i z t} \int D(q, \bar{q}) L_{n}\left(\alpha, z ; \bar{q}, q^{\prime}\right) e^{-i t a(\bar{q})} d \bar{q}
\end{array}
$$

wherefrom

$$
\begin{aligned}
& \partial_{z}^{k} \partial_{q}^{\alpha} \partial_{q^{\prime}}^{\beta} L_{n+1}\left(\alpha, z ; q, q^{\prime}\right)= \\
& i \alpha^{2} \int_{0}^{\infty} d t(i t)^{k} e^{i z t} \int \partial_{q}^{\alpha} D(q, \bar{q}) \partial_{q^{\prime}}^{\beta} L_{n}\left(\alpha, z ; \bar{q}, q^{\prime}\right) e^{-i t a(\bar{q})} d \bar{q}
\end{aligned}
$$

Using (4.3), the induction hypothesis and the condition 1 for $a(q)$, the internal integral can be represented by the stationary phase method as

$$
\hat{C} \frac{\partial_{q}^{\alpha} D\left(q, \bar{q}_{0}\right) \partial_{q^{\prime}}^{\beta} L_{n}\left(\alpha, z ; \bar{q}_{0}, q^{\prime}\right) e^{-i t a\left(\bar{q}_{0}\right)}}{t^{d / 2}+1}+\Delta_{\alpha \beta}\left(t ; q, q^{\prime}\right),
$$

where $\hat{C}$ is an absolute constant, and the kernel $\Delta_{\alpha \beta}$ is bounded by

$$
\left|\Delta_{\alpha \beta}\left(t ; q, q^{\prime}\right)\right| \leq \bar{N}\left(C \alpha^{2}\right)^{n-1}\|h\|_{L_{2}}^{2} \frac{h(q) h\left(q^{\prime}\right)}{t^{d / 2+1}+1}
$$

with some constant $\bar{N}$ dependent on $d$ and on the function $a$. The integral

$$
\int_{0}^{\infty} d t \frac{(i t)^{k}}{t^{d / 2}+1} e^{i\left(z-a\left(\bar{q}_{0}\right)\right) t}
$$

is absolutely convergent for all $k \leq s$ and defines a continuous function of $z$ in $\mathfrak{I m} z \geq 0$, which, for $k=s$, is Hölder continuous with respect to this variable. We have that the contribution to (4.15) of the first term in (4.16) has the estimate

$$
\begin{array}{r}
\left|i \alpha^{2} \hat{C} \int_{0}^{\infty} d t(i t)^{k} e^{i t\left(z-a\left(\bar{q}_{0}\right)\right)}\left(t^{d / 2}+1\right)^{-1} \partial_{q}^{\alpha} D\left(q, \bar{q}_{0}\right) \partial_{q^{\prime}}^{\beta} L_{n}\left(\alpha, z ; \bar{q}_{0}, q^{\prime}\right)\right| \\
\leq \tilde{C} h\left(\bar{q}_{0}\right)^{2} \tilde{N}\left(C \alpha^{2}\right)^{n-1} h(q) h\left(q^{\prime}\right)
\end{array}
$$

where $\tilde{C}$ is a constant. One proves in the same way the Hölder condition (4.8) for $k=s$. A similar estimate holds for the integral of the second term in (4.16):

$$
\left|\int_{0}^{\infty}(i t)^{k} e^{i z t} \Delta_{\alpha \beta}\left(t ; q, q^{\prime}\right) d t\right| \leq \tilde{C}\|h\|_{L_{2}}^{2} \bar{N}\left(C \alpha^{2}\right)^{n-1} h(q) h\left(q^{\prime}\right) .
$$

By taking $C=\max \left\{N, \tilde{C}\left(\left|h\left(\bar{q}_{0}\right)\right|^{2} \tilde{N}+\|h\|_{L_{2}}^{2} \bar{N}\right)\right\}$, one gets the estimate (4.12), the existence of the limit (4.13) and the assertion (iii) for $n$ replaced by $n+1$. 
Once we have $R_{B}(z)$, it is an easy matter to write down the solution of equation (4.1) for $z \in \mathbb{C} \backslash I$ as

$$
f_{1}=R_{B}(z)\left[g_{1}-\alpha f_{0} v\right]
$$

where

$$
f_{0}=\frac{1}{\Delta(z)}\left[g_{0}-\alpha\left(v, R_{B}(z) g_{1}\right)\right]
$$

whenever $\Delta(z) \neq 0$. Here,

$$
\Delta(z)=e^{(0)}-z-\alpha^{2}\left(v, R_{B}(z) v\right)
$$

Clearly, $\Delta(z)$ is analytic in $\mathbb{C} \backslash I$, has limits at the cut $I$ :

$$
\lim _{\varepsilon \searrow 0} \Delta(x \pm i \varepsilon)=\Delta^{ \pm}(x), x \in I
$$

and the limits $\Delta^{ \pm}(x)$ are $s+1 / 3$ times differentiable, by the same reasoning as in Lemma 4.1. More precisely,

$$
\left|\frac{d^{k}}{d x^{k}}\left(\Delta^{ \pm}(x)+x\right)\right| \leq \text { const }, k=0, \ldots, s+1 / 3 .
$$

As one can read from equations (4.19), (4.20), the continuous spectrum of the operator $A$ equals the interval $I$. Besides, the real zeroes of $\Delta(z)$ below $\bar{a}$, if any, are eigenvalues of $A$. Since $-\alpha^{2}\left(v, R_{B}(x) v\right)$ is decreasing for $x<\bar{a}, \Delta(x)$ is strictly decreasing from $+\infty$ to $\Delta(\bar{a})$ on this interval, therefore $A$ has one simple eigenvalue $e<\bar{a}$ with eigenvector $\psi_{0}=\left(f_{0}, f_{1}=-\alpha f_{0} R_{B}(e) v\right) \in \mathcal{H}^{(\leq 1)}$, if, and only if, $\Delta(\bar{a})<0$. As, for small $\alpha$

$$
\pm \mathfrak{I m} \Delta^{ \pm}(x)=\alpha^{2} \pi \int_{a(q)=x}|v(q)|^{2} d q+0\left(\alpha^{4}\right)>0, x \in I
$$

in view of condition 3 , it follows that there are no eigenvalues of $A$ embedded in the continuous spectrum (see [7]).

Remark 4.3 It is easy to show using the explicit formulae for $R_{A}(z)$ that the general criteria of the absence of the singular continuous spectrum [8] are met in our case, hence that the continuous spectrum I is absolutely continuous. Therefore, we have

$$
\mathcal{H}^{(\leq 1)}= \begin{cases}\mathcal{H}^{a c}, & \Delta(\bar{a}) \geq 0 \\ \left\{c \psi_{0}\right\} \oplus \mathcal{H}^{a c}, & \Delta(\bar{a})<0\end{cases}
$$

We come now to the scattering theory for the pair of self-adjoint operators $\left(A, B_{0}\right)$, where we denoted $B_{0}$ the operator of multiplication with $a(q)$ acting in $\mathcal{H}^{(1)}=L^{2}\left(\mathbf{R}^{d}, d q\right)$. We denote $E: \mathcal{H}^{(1)} \rightarrow \mathcal{H}^{(\leq 1)}$ the injection $E \varphi=(0, \varphi) \in \mathcal{H}^{(\leq 1)}, \varphi \in \mathcal{H}^{(1)}$. Known existence criteria for the wave operators (see e.g. [8]) can be applied to our case and ensure the existence of the strong limit:

$$
s-\lim _{t \rightarrow \infty} e^{i t A} E e^{-i t B_{0}}=\Omega^{+},
$$


which is a unitary operator $\Omega^{+}: \mathcal{H}^{(1)} \rightarrow \mathcal{H}^{a c} \subset \mathcal{H}^{(\leq 1)}$. The generalized eigenfunctions of the operator $B_{0}$ are $\delta_{q}(\cdot)=\delta(q-\cdot)$, therefore, using known formulae in scattering theory, one can take

$$
\psi^{q}=\Omega^{+} \delta_{q}=\lim _{\varepsilon \searrow 0} i \varepsilon R_{A}(a(q)-i \varepsilon) E \delta_{q}
$$

as generalized eigenvectors of $A$ corresponding to the eigenvalue $a(q)$. Explicitly, in view of (4.19), (4.20) and Lemma 4.1, one gets for $\psi^{q}=\left(f_{0}^{q}, f_{1}^{q}(\cdot)\right)$ the following expressions:

$$
\begin{aligned}
& f_{0}^{q}=-\frac{\alpha}{\Delta^{-}(a(q))}\left[v(q)+\alpha^{2} \int \frac{K^{-}\left(\alpha, a(q) ; q^{\prime}, q\right) \bar{v}\left(q^{\prime}\right) d q^{\prime}}{a\left(q^{\prime}\right)-a(q)+i 0}\right], \\
& f_{1}^{q}\left(q^{\prime}\right)=\delta\left(q-q^{\prime}\right)+\frac{\alpha^{2} K^{-}\left(\alpha, a(q) ; q^{\prime}, q\right)}{a\left(q^{\prime}\right)-a(q)+i 0}- \\
& \alpha f_{0}^{q} \frac{1}{a\left(q^{\prime}\right)-a(q)+i 0}\left[v\left(q^{\prime}\right)+\alpha^{2} \int \frac{K^{-}\left(\alpha, a(q) ; q^{\prime}, q^{\prime \prime}\right) \bar{v}\left(q^{\prime \prime}\right) d q^{\prime \prime}}{a\left(q^{\prime \prime}\right)-a(q)+i 0}\right] .
\end{aligned}
$$

This somewhat formal derivation of the formulas (4.25), (4.26) will be justified by the next lemma, which proves that $\psi^{q} \in \mathbb{C} \oplus \mathcal{S}^{\prime}\left(\mathbb{R}^{d}\right)$ (where $\mathcal{S}^{\prime}\left(\mathbb{R}^{d}\right)$ is the space of tempered distributions) and that it verifies the intertwining property of the wave-operator $\Omega^{+}$.

Lemma 4.4 Let $d \geq 3$. Then

1. For every fixed $q \in \mathbb{R}^{d}$, $f_{0}^{q}$ is finite and it is bounded and continuous as a function of $q$.

2. For every fixed $q \in \mathbf{R}^{d}, f_{1}^{q}(\cdot) \in \mathcal{S}^{\prime}\left(\mathbb{R}^{d}\right)$; moreover, for every fixed $q^{\prime} \in \mathbb{R}^{d}, f_{1}^{q}\left(q^{\prime}\right) \in$ $\mathcal{S}^{\prime}\left(\mathbb{R}^{d}\right)$ with respect to $q$.

3. For $\varphi \in \mathcal{S}\left(\mathbb{R}^{d}\right)$, let us consider the vector $\psi_{\varphi}=\left(C_{\varphi, 0}, C_{\varphi, 1}(\cdot)\right) \in \mathcal{H}^{(\leq 1)}$, where

$$
\begin{aligned}
C_{\varphi, 0} & =\int f_{0}^{q} \varphi(q) d q, \\
C_{\varphi, 1}\left(q^{\prime}\right) & =\int f_{1}^{q}\left(q^{\prime}\right) \varphi(q) d q .
\end{aligned}
$$

Then, for any $\varphi_{1}, \varphi_{2} \in \mathcal{S}\left(\mathbb{R}^{d}\right)$,

$$
\left(\psi_{\varphi_{1}}, \psi_{\varphi_{2}}\right)_{\mathcal{H}(\leq 1)}=\bar{C}_{\varphi_{1}, 0} C_{\varphi_{2}, 0}+\int \bar{C}_{\varphi_{1}, 1}(q) C_{\varphi_{2}, 1}(q) d q=\left(\varphi_{1}, \varphi_{2}\right)_{L_{2}}
$$

therefore the application $\varphi \mapsto \psi_{\varphi}$ extends to an isometry $\Omega^{+}: L^{2}\left(\mathbb{R}^{d}, d q\right) \rightarrow \mathcal{H}^{(\leq 1)}$. 4. The range of the operator $\Omega^{+}$is $\mathcal{H}^{a c}$ and $A \Omega^{+}=\Omega^{+} B_{0}$.

Remark 4.5 The relation (4.29) may be written in the following formal way

$$
\left(\psi^{q}, \psi^{q^{\prime}}\right)_{\mathcal{H}^{(\leq 1)}}=\bar{f}_{0}^{q} f_{0}^{q^{\prime}}+\left(f_{1}^{q}, f_{1}^{q^{\prime}}\right)_{\mathcal{H}^{(1)}}=\delta\left(q-q^{\prime}\right)
$$

meaning the orthonormality of the generalized functions $\left\{\psi^{q}, q \in \mathbb{R}^{d}\right\}$. 
Remark 4.6 Usually, the generalized eigenvectors of a self-adjoint operator $A$ acting in the Hilbert space $\mathcal{H}$ are defined as derivatives $d E_{\lambda} \varphi / d \sigma_{\varphi}(\lambda)$, where $\varphi \in \mathcal{H}$ is an arbitrary vector, $\left\{E_{\lambda}\right\}$ is the family of spectral projections of $A$, and $\sigma_{\varphi}(\lambda)$ is the spectral measure corresponding to $\varphi$. Moreover, if $A$ leaves invariant a certain dense linear subspace $\mathcal{H}_{+} \subset$ $\mathcal{H}$ and $\mathcal{H}_{+}$has a Hilbert space structure such that the inclusion is quasi-nuclear, then the derivative $d E_{\lambda} \varphi / d \sigma_{\varphi}(\lambda)=\chi_{\lambda}$ exists as an element of the conjugate space $\mathcal{H}_{-}=\mathcal{H}_{+}^{*}$ and it is an eigenvector with eigenvalue $\lambda$ of the adjoint: $\left(\left.A\right|_{\mathcal{H}_{+}}\right)^{*}$, of the restriction of $A$ to $\mathcal{H}_{+}$, which is an extension of $A$. The vectors $\chi_{\lambda} \in \mathcal{H}_{-}$are usually called generalized eigenvectors of the operator $A$. It can be shown that the generalized vectors introduced above are generalized eigenvectors of $A$ in this sense. The same remark is valid for the generalized eigenvectors of the operator $H_{p}$ (which will be constructed farther on).

Proof of Lemma 4.4

1. This assertion follows easily from the representation

$$
\begin{aligned}
& \int K^{-}\left(\alpha, a(q) ; q^{\prime}, q\right) \bar{v}\left(q^{\prime}\right)\left[a\left(q^{\prime}\right)-a(q)+i 0\right]^{-1} d q^{\prime}= \\
& i \int_{0}^{\infty} d t \int e^{i t\left(a\left(q^{\prime}\right)-a(q)\right)} K^{-}\left(\alpha, a(q) ; q^{\prime}, q\right) \bar{v}\left(q^{\prime}\right) d q^{\prime}
\end{aligned}
$$

by applying the stationary phase method as done already in the proof of Lemma 4.1 2 . In order to prove the second assertion, we have to consider $C_{\varphi, 1}\left(q^{\prime}\right)$. To this aim, we represent the $q^{\prime \prime}$-integral in (4.26) ) as before, using the stationary phase method:

$$
\begin{aligned}
& I\left(x ; q^{\prime}\right):=i \int_{0}^{\infty} d t \int e^{i t\left(a\left(q^{\prime \prime}\right)-x\right)} K^{-}\left(\alpha, x ; q^{\prime}, q^{\prime \prime}\right) \bar{v}\left(q^{\prime \prime}\right) d q^{\prime \prime} \\
& =i \int_{0}^{\infty} d t\left[\frac{\hat{C}}{t^{d / 2}+1} e^{i t\left(a\left(\bar{q}_{0}\right)-x\right)} K^{-}\left(\alpha, x ; q^{\prime}, \bar{q}_{0}\right) \bar{v}\left(\bar{q}_{0}\right)+\Delta\left(x ; q^{\prime}, t\right)\right],
\end{aligned}
$$

where the correction term $\Delta$ satisfies the estimates

$$
\left|\partial_{x}^{k} \partial_{q}^{\alpha} \Delta\left(x ; q^{\prime}, t\right)\right| \leq \frac{\hat{N} h\left(q^{\prime}\right)}{t^{d / 2+1}+1}
$$

for all multi-indices $\alpha,|\alpha| \leq[d / 2]+1$, and $k=0, \ldots, s+1 / 3$, where $\hat{N}$ is a constant. Hence, $I\left(x ; q^{\prime}\right)$ fulfills for $d \geq 3$ the estimates

$$
\left|\partial_{x}^{k} \partial_{q}^{\alpha} I\left(x ; q^{\prime}\right)\right| \leq \tilde{N} h\left(q^{\prime}\right) ;|\alpha| \leq[d / 2]+1, k=0, \ldots, s+1 / 3 .
$$

The contribution of this term to $C_{\varphi, 1}\left(q^{\prime}\right)$ is:

$$
\begin{aligned}
& \int d q \int d q^{\prime \prime} \frac{f_{0}^{q} \varphi(q) K^{-}\left(\alpha, a(q) ; q^{\prime}, q^{\prime \prime}\right) \bar{v}\left(q^{\prime \prime}\right)}{\left(a\left(q^{\prime}\right)-a(q)+i 0\right)\left(a\left(q^{\prime \prime}\right)-a(q)+i 0\right)} \\
& =\int d q \frac{f_{0}^{q} \varphi(q) I\left(a(q) ; q^{\prime}\right)}{a\left(q^{\prime}\right)-a(q)+i 0}=\int_{\mathbb{R}} d x \frac{m(x) I\left(x ; q^{\prime}\right)}{a\left(q^{\prime}\right)-x+i 0},
\end{aligned}
$$


where

$$
m(x)=\int_{a(q)=x} f_{0}^{q} \varphi(q) d q .
$$

As it follows from the proof of the point $1, m(x)$ is $s+1 / 3$ times differentiable. The same property is shared by $I\left(x ; q^{\prime}\right)$ as a function of $x$ for every fixed $q^{\prime}$. Therefore, the integral over $x$ in (4.32) converges. The convergence of the other terms entering $C_{\varphi, 1}\left(q^{\prime}\right)$ can be proved similarly.

3. Using the representation

$$
\varphi(q)=\int \varphi\left(q_{0}\right) \delta\left(q-q_{0}\right) d q_{0}, \varphi \in \mathcal{S}\left(\mathbb{R}^{d}\right)
$$

and the formula (4.24) we find that

$$
\Omega^{+} \varphi=\int \varphi\left(q_{0}\right) \psi^{q_{0}} d q_{0}=\left(C_{\varphi, 0}, C_{\varphi, 1}(\cdot)\right) \in \mathcal{H}^{a c} \subseteq \mathcal{H}^{(\leq 1)}
$$

In view of the unitarity of the application $\Omega^{+}: L^{2}\left(\mathbb{R}^{d}\right) \rightarrow \mathcal{H}^{a c}$, one has

$$
\begin{aligned}
& \left(\varphi_{1}, \varphi_{2}\right)_{L^{2}\left(\mathbb{R}^{d}\right)}=\left(\Omega^{+} \varphi_{1}, \Omega^{+} \varphi_{2}\right) \\
& =\bar{C}_{\varphi_{1}, 0} C_{\varphi_{2}, 0}+\int \bar{C}_{\varphi_{1}, 1}(q) C_{\varphi_{2}, 1}(q) d q .
\end{aligned}
$$

4. Since $\mathcal{S}\left(\mathbb{R}^{d}\right)$ is dense in $L^{2}\left(\mathbb{R}^{d}\right)$, the image $\Omega^{+} \mathcal{S}\left(\mathbf{R}^{d}\right)$ is dense in $\mathcal{H}^{a c}$, therefore, in view of the unitarity of $\Omega^{+}, \Omega^{+} L^{2}\left(\mathbf{R}^{d}\right)=\mathcal{H}^{a c}$. The intertwining property $A \Omega^{+}=\Omega^{+} B$ is obtained in the standard way. Lemma 4.4 is proved.

This lemma implies in particular that any vector $\psi \in \mathcal{H}^{a c}$ has a unique representation as

$$
\psi=\psi_{f}=\int_{\mathbb{R}^{d}} f\left(q_{0}\right) \psi^{q_{0}} d q_{0}:=\lim _{\varphi_{n} \rightarrow f} \psi_{\varphi_{n}}, f \in L^{2}\left(\mathbb{R}^{d}\right) .
$$

Here, the limit in the right-hand side is meant in $\mathcal{H}^{a c}$ and $\left\{\varphi_{n}\right\}$ is a sequence of elements of $\mathcal{S}\left(\mathbb{R}^{d}\right)$ converging to $f$ in $L_{2}$.

\subsection{Construction of the one-boson subspace}

As explained in Section 2, the construction of the one-boson subspace of $H_{p}$ relies on the spectral representation of the operators $\left\{A_{p}(\xi)\right\}_{\xi \leq \kappa}$, see (2.10), entering the reduced system (3.25):

$$
\begin{aligned}
\left(A_{p}(\xi) F\right)_{0}=e_{0, p}^{0} f_{0} & +\alpha \int \overline{c(p-q, q)} f_{1}(q) d q \\
\left(A_{p}(\xi) F\right)_{1}(q)=\alpha c(p-q, q) f_{0} & +a_{p}(\xi ; q) f_{1}(q) \\
& +\alpha^{2} \int D_{p}\left(\xi ; q, q^{\prime}\right) f_{1}\left(q^{\prime}\right) d q^{\prime} \\
& F=\left(f_{0}, f_{1}(\cdot)\right) \in \mathcal{H}^{(\leq 1)} .
\end{aligned}
$$


Since for any $\xi \leq \kappa$ the operator $A_{p}(\xi)$ satisfies all the assumptions of the previous subsection, there exists a family

$$
\left\{F_{\xi, 1}^{q}=\left(f_{\xi, 0}^{q}, f_{\xi, 1}^{q}(\cdot)\right)\right\}_{q \in \mathbb{R}^{d}}
$$

of generalized eigenvectors of $A_{p}(\xi)$ with eigenvalues $\left\{a_{p}(\xi ; q)\right\}_{q \in \mathbb{R}^{d}}$, given by (4.25), (4.26), where $a(q)$ is replaced by $a_{p}(\xi ; q)$, and $\Delta^{-}, K^{-}$by the functions $\Delta_{\xi}^{-}, K_{\xi}^{-}$, entering the expression of the resolvent of $A_{p}(\xi)$. Let $F_{\xi, 2}^{q}$ be constructed in terms of $f_{\xi, 1}^{q}(\cdot)$ according to (3.9), i.e. $F_{\xi, 2}^{q}=S(\xi) f_{\xi, 1}^{q}$ where the application $S(\xi)$ was introduced in equation (3.8) (more precisely, $S(\xi)$ is the extension of that operator to the space $\mathcal{B}_{1}^{(k)}$ defined below), where the coefficient functions are the solution of the fixed point equation (3.14). Then, the complete sequence

$$
F_{\xi}^{q}=\left(F_{\xi, 1}^{q}, F_{\xi, 2}^{q}\right)=\left(f_{\xi, 0}^{q}, f_{\xi, 1}^{q}\left(q_{1}\right), f_{\xi, 2}^{q}\left(q_{1}, q_{2}\right), \ldots\right)
$$

satisfies the equation

$$
H_{p} F_{\xi}^{q}=\xi F_{\xi}^{q}+\left(a_{p}(\xi ; q)-\xi\right) \hat{F}_{\xi}^{q},
$$

where we denoted $\hat{F}_{\xi}^{q}=\left(F_{\xi, 1}^{q}, 0\right)$. Therefore, if $\xi(q)$ is a solution of equation

$$
a_{p}(\xi ; q)-\xi=0
$$

then $F_{\xi(q)}^{q}$ is a generalized eigenvector of the operator $H_{p}$ with eigenvalue $\xi(q) \equiv \xi_{p}(q)$, cf (2.13) in Remark 2.1]

We shall give below sense to the generalized eigenfunctions $F_{\xi}^{q}$ as elements of the dual $\left(\mathcal{B}^{(k)}\right)^{\prime}$ of an auxiliary Banach space $\mathcal{B}^{(k)}, k=[d / 2]+2$, densely and continuously embedded in the Fock space $\mathcal{F}$.

Definition 4.7 Let us denote $\mathcal{B}_{n}^{(k)}$ the space of all symmetric functions $g$ of $n$ variables $q_{1}, \ldots, q_{n} \in \mathbb{R}^{d}, k$ times continuously differentiable with respect to each $q_{i}$, and for which there exists a constant $C$ such that, for all multi-indices $\alpha=\left(\alpha_{1}, \ldots, \alpha_{n}\right), \alpha_{i}=\left(\alpha_{i}^{1}, \ldots, \alpha_{i}^{d}\right)$, $\left|\alpha_{i}\right|=\sum_{s=1}^{d} \alpha_{i}^{s} \leq k$, one has

$$
\left|\partial_{q}^{\alpha} g\left(q_{1}, \ldots, q_{n}\right)\right| \leq C \prod_{i=1}^{n} h\left(q_{i}\right), \forall q_{1}, \ldots, q_{n} \in \mathbb{R}^{d}
$$

It is a Banach space if endowed with the norm $\|g\|_{n}^{(k)}=\inf C$, where the infimum is taken over all $C$ for which the estimate 14.41) holds.

Clearly, the inclusion $\mathcal{B}_{n}^{(k)} \subset \mathcal{H}^{(n)}$ is continuous and $\mathcal{B}_{n}^{(k)}$ is dense in $\mathcal{H}^{(n)}$. Let next

$$
\mathcal{B}^{(k)}=\mathbb{C}+\mathcal{B}_{1}^{(k)}+\ldots+\mathcal{B}_{n}^{(k)}+\ldots \subset \mathcal{F}
$$


be the space of sequences

$$
G=\left(g_{0}, g_{1}\left(q_{1}\right), \ldots, g_{n}\left(q_{1}, \ldots, q_{n}\right), \ldots\right), g_{0} \in \mathbb{C}, g_{n} \in \mathcal{B}_{n}^{(k)},
$$

with norm

$$
\|G\|_{\mathcal{B}^{(k)}}^{(k)}=\left[\left|g_{0}\right|^{2}+\sum_{n \geq 1} \frac{1}{n !}\left(\left\|g_{n}\right\|_{n}^{(k)}\right)^{2}\right]^{1 / 2} .
$$

Obviously, $\mathcal{B}^{(k)}$ is continuously and densely embedded in the Fock space $\mathcal{F}$, as required. The dual $\left(\mathcal{B}^{(k)}\right)^{\prime}$ of $\mathcal{B}^{(k)}$ consists of sequences $F=\left(f_{0}, f_{1}, \ldots, f_{n}, \ldots\right)$, where $f_{0} \in \mathbb{C}$, and $f_{n} \in\left(\mathcal{B}_{n}^{(k)}\right)^{\prime}$ are linear continuous functionals on $\mathcal{B}_{n}^{(k)}$; thereby, the value of $F$ at an element $G \in \mathcal{B}^{(k)}$ is given by the series:

$$
(F, G)=\left[\bar{f}_{0} g_{0}+\sum_{n \geq 1} \frac{1}{n !}\left(f_{n}, g_{n}\right)\right]^{1 / 2}
$$

and the norm of $F$ is

$$
\|F\|_{\left(\mathcal{B}^{(k)}\right)^{\prime}}^{(k)}=\left[\left|f_{0}\right|^{2}+\sum_{n \geq 1} \frac{1}{n !}\left(\left\|f_{n}\right\|_{\left(\mathcal{B}_{n}^{(k)}\right)^{\prime}}\right)^{2}\right]^{1 / 2} .
$$

Clearly, $\mathcal{F} \subset\left(\mathcal{B}^{(k)}\right)^{\prime}$ and the inclusion is continuous.

Lemma 4.8 For every $q \in \mathbb{R}^{d}$ and $\xi \leq \kappa, F_{\xi}^{q} \in\left(\mathcal{B}^{(k)}\right)^{\prime}$ and has the representation

$$
F_{\xi}^{q}=\hat{\delta}_{q}+\tilde{F}_{\xi}^{q}
$$

where $\hat{\delta}_{q}=\left(0, \delta_{q}, 0, \ldots\right)$ and

$$
\left\|\tilde{F}_{\xi}^{q}\right\|_{\left(\mathcal{B}^{(k)}\right)^{\prime}} \leq M \alpha h(q)
$$

for some constant $M$.

Proof: We prove this statement in three steps.

I. The $n=0$ component of $\tilde{F}_{\xi}^{q}, \tilde{f}_{\xi, 0}^{q}$, is given by equation (4.25), where $v(q)=c(p-q, q)$, $a(q)=a_{p}(\xi, q)$ and $K^{-}=K_{\xi}^{-}$. If condition 2 in Section 4.1 is fulfilled for every $\xi \leq \kappa$ and $p$, we have $\Delta^{-}(a(q)) \geq \tau>0$, therefore we obtain for the first term in (4.25)

$$
\left|\frac{(-\alpha v(q))}{\Delta^{-}(a(q))}\right| \leq \frac{\alpha}{\tau} h(q) .
$$

The second term in (4.25) is treated using as before the stationary phase method, which gives

$$
\begin{aligned}
& \int K^{-}\left(\alpha, a(q) ; q^{\prime}, q\right) \bar{v}\left(q^{\prime}\right)\left(a\left(q^{\prime}\right)-a(q)+i 0\right)^{-1} d q^{\prime} \\
& =i \int_{0}^{\infty} d t\left[\hat{C}\left(t^{d / 2}+1\right)^{-1} e^{i t\left(a\left(\bar{q}_{0}\right)-a(q)\right)} K^{-}\left(\alpha, a(q) ; \bar{q}_{0}, q\right) \bar{v}\left(\bar{q}_{0}\right)+\Delta(q, t)\right]
\end{aligned}
$$


where

$$
|\Delta(q, t)| \leq C h(q)\left(t^{d / 2+1}+1\right)^{-1}
$$

for some constant $C$. Equations (4.48), (4.49), (4.50) provide

$$
\left|\tilde{f}_{\xi, 0}^{q}\right| \leq B \alpha h(q)
$$

II. The $n=1$ component of $F_{\xi}^{q}, f_{\xi, 1}^{q}=\delta_{q}+\tilde{f}_{\xi, 1}^{q}$, is given by equation (4.26), with the same assignments for $v, a$, and $K^{-}$. Again, reducing the estimate of every integral entering $\left(\tilde{f}_{\xi, 1}^{q}, g_{1}\right)$ for a generic $g_{1} \in \mathcal{B}_{1}^{(k)}$ to the estimate of the corresponding oscillatory integral, and using thereby the estimate (4.48), we obtain

$$
\left\|\tilde{f}_{\xi, 1}^{q}\right\|_{\left(\mathcal{B}_{1}^{(k)}\right)^{\prime}} \leq L \alpha^{2} h(q)
$$

III. The higher components of $\tilde{F}_{\xi}^{q},\left\{\tilde{f}_{\xi, n}^{q}\right\}_{n \geq 2}$, are estimated using their representation (3.9) in terms of $f_{\xi, 1}^{q}$. We have

$$
\begin{aligned}
& \left(\tilde{f}_{\xi, n}^{q}, g_{n}\right)= \\
& =\sum_{i=1}^{n} \int b_{n}\left(q_{1}, \ldots, \check{q}_{i} . ., q_{n} ; q_{i}\right) f_{\xi, 1}^{q}\left(q_{i}\right) g_{n}\left(q_{1}, \ldots, q_{n}\right) d q_{1} \ldots d q_{n} \\
& +\int d_{n}\left(q_{1}, \ldots, q_{n} ; q^{\prime}\right) f_{\xi, 1}^{q}\left(q^{\prime}\right) g_{n}\left(q_{1}, \ldots, q_{n}\right) d q_{1} \ldots d q_{n} d q^{\prime}
\end{aligned}
$$

Using the estimates for $b_{n}, d_{n}$ and their derivatives (see (3.12) and Lemma 3.2), and also the bound (4.52), we have that

$$
\begin{aligned}
& \int\left|\int b_{n}\left(q_{1}, \ldots, \check{q}_{i} . ., q_{n} ; q_{i}\right) \tilde{f}_{\xi, 1}^{q}\left(q_{i}\right) g_{n}\left(q_{1}, \ldots, q_{n}\right) d q_{i}\right| d q_{1} \ldots d \check{q}_{i} \ldots d q_{n} \\
& \leq C_{1} \alpha\left(\lambda_{2}(p)-\kappa\right)^{-1}\left\|g_{n}\right\|_{\mathcal{B}_{n}^{(k)}}\left(1+L \alpha^{2}\right) h(q)\left(\int h\left(q^{\prime}\right) d q^{\prime}\right)^{n-1}
\end{aligned}
$$

for $i=1, \ldots, n$, and also that

$$
\begin{aligned}
& \left|\int d_{n}\left(q_{1}, \ldots, q_{n} ; q^{\prime}\right) \tilde{f}_{\xi, 1}^{q}\left(q^{\prime}\right) g_{n}\left(q_{1}, \ldots, q_{n}\right) d q_{1} \ldots d q_{n} d q\right| \\
& \leq C_{2} \alpha\left(\lambda_{2}(p)-\kappa\right)^{-1}\left\|g_{n}\right\|_{\mathcal{B}_{n}^{(k)}}\left(1+L \alpha^{2} h(q)\right) h(q)\left(\int h\left(q^{\prime}\right) d q^{\prime}\right)^{n} .
\end{aligned}
$$

Hence, with suitable constants $\tilde{C}, \tilde{L}$,

$$
\left\|\tilde{f}_{\xi, n}^{q}\right\|_{\left(\mathcal{B}_{n}^{(k)}\right)^{\prime}} \leq \tilde{C} \frac{\alpha}{\lambda_{2}(p)-\kappa}\left(1+\tilde{L} \alpha^{2}\right) \cdot h(q)\left(\int h\left(q^{\prime}\right) d q^{\prime}\right)^{n-1} .
$$

Putting together equations (4.51), (4.52) and (4.54), we obtain (4.47). The lemma is proved.

Now we come back to the study of the generalized eigenvectors $F_{\xi(q)}^{q}$. 
Let us remark that $a_{p}(\xi ; q)$ is, for every fixed $q$, a smooth, monotonously decreasing function of $\xi$ on $(-\infty, \kappa]$. If

$$
G_{p}^{(1), \kappa}=\left\{q \in \mathbb{R}^{d}: a_{p}(\kappa ; q)-\kappa<0\right\},
$$

then equation (4.40) has a unique solution $\xi(q)<\kappa$ if $q \in G_{p}^{(1), \kappa}$, and no solution if $q \notin G_{p}^{(1), \kappa}$, see Figure 1 .

Proposition 4.9 The function $\xi(q) \equiv \xi_{p}(q)$ can be represented in the form

$$
\xi_{p}(q)=\varepsilon(q)+\gamma(p-q)
$$

where the function $\gamma(k)$ is defined in the domain $\left\{k: p-k \in G_{p}^{(1), \kappa}\right\}$.

Proof: Indeed, let us use the expansion

$$
\mu=\mu_{0}-\Gamma \mu_{0}+\Gamma^{2} \mu_{0}+\ldots
$$

for the solution of equation (3.14), and remark that the function $b_{2}^{0}\left(q_{1} ; q\right)$ appearing in (3.15) can be written in the form

$$
b_{2}^{0}\left(q_{1} ; q\right) \equiv b_{2, p}^{0}\left(q_{1} ; q\right)=\varphi_{q_{1}}^{0}(p-q ; z-\varepsilon(q))
$$

with $\varphi_{q_{1}}^{0}(k ; w)$ defined for $k \in \mathbb{R}^{d}, w \in \mathbb{C}$ such that $\mathfrak{R e} w<\kappa-\varepsilon(q)$. One can prove by induction, using the formula (3.10), that, in every term $\Gamma^{k} \mu_{0}$ of the expansion (4.57), the function $b_{n, p}^{(k)}\left(z ; q_{1}, \ldots, q_{n-1} ; q\right)$ has, for fixed $q_{1}, \ldots, q_{n-1}$, a form similar to (4.58), i.e.

$$
b_{n, p}^{(k)}\left(z ; q_{1}, \ldots, q_{n-1} ; q\right)=\varphi_{q_{1}, \ldots, q_{n-1}}^{(k)}(p-q ; z-\varepsilon(q)) .
$$

Then, it follows that the coefficient functions $b_{n} \equiv b_{n, p}$ of the solution $\mu$ given by (4.57) are of the same form (4.59), in particular,

$$
b_{2, p}\left(q_{1} ; q\right)=\varphi_{q_{1}}(p-q ; z-\varepsilon(q))
$$

Plugging this expression into (3.23), we find that $m_{p}$ depends only on the differences $p-q$ and $z-\varepsilon(q)$ :

$$
m_{p}(z, q)=\tau(p-q ; z-\varepsilon(q)) .
$$

Hence, by virtue of (1.10), (3.26) and (4.61) the equation (4.40)

$$
\frac{1}{2}(p-q)^{2}+\varepsilon(q)+m_{p}(\xi, q)=\xi
$$

writes as

$$
\frac{1}{2}(p-q)^{2}+\tau(p-q ; \gamma)=\gamma
$$

This implies that $\gamma \equiv \xi-\varepsilon(q)$ is a function of $p-q$ alone. 
Clearly, by the convexity of $e_{1, p}^{0}$ and the asymptotical properties of $m_{p}(\xi, q)$ given in Corollary 3.4 $G_{p}^{(1), \kappa}$ is a bounded domain, nonvoid for $\kappa>\lambda_{1}(p)$, and $\underset{q \in G_{p}^{(1), \kappa}}{\min } \xi(q)=\lambda_{1}(p)$.

By the smoothness of $a_{p}(\xi, q)$ with respect to both arguments, the function $\xi(q)$ defined on $G_{p}^{(1), \kappa}$ is also smooth. Moreover, for $\alpha$ sufficiently small, this function has a unique critical point (namely, a minimum), which is nondegenerate. In particular, it follows that on every level of $\xi(q)$,

$$
\chi_{x}=\left\{q \in G_{p}^{(1), \kappa}: \xi(q)=x\right\},
$$

one can define a measure $\nu_{x}$ (the Gelfand-Leray measure, see [9]), such that, for any integrable function $\varphi$ on $G_{p}^{(1), \kappa}$,

$$
\int_{G_{p}^{(1), \kappa}} \varphi(q) d q=\int_{\lambda_{1}(p)}^{\kappa} d x \int_{\chi_{x}} \varphi_{x} d \nu_{x},
$$

where $\varphi_{x}=\left.\varphi\right|_{\chi_{x}}$ is the restriction of $\varphi$ to the surface $\chi_{x}$. From (4.64) it follows that $L^{2}\left(G_{p}^{(1), \kappa}, d q\right)$ can be represented as a direct integral of Hilbert spaces:

$$
L^{2}\left(G_{p}^{(1), \kappa}, d q\right)=\int_{\left[\lambda_{1}(p), \kappa\right]}^{\oplus} \mathcal{H}_{x} d x,
$$

with $\mathcal{H}_{x}:=L^{2}\left(\chi_{x}, \nu_{x}\right)$.

Let us now consider the family $\left\{F_{\xi(q)}^{q}\right\}_{q \in G_{p}^{(1), \kappa}} \subset\left(\mathcal{B}^{(k)}\right)^{\prime}$ of generalized eigenvectors of $H_{p}$. The next lemma, which may be stated formally as an approximate orthonormality of this family, is an important element of our constructions. We denote

$$
F(\varphi):=\int_{G_{p}^{\kappa}} F_{\xi(q)}^{q} \varphi(q) d q \in\left(\mathcal{B}^{(k)}\right)^{\prime},
$$

for $\varphi \in \mathcal{D}\left(G_{p}^{(1), \kappa}\right)$, the space of infinitely differentable functions with support in $G_{p}^{(1), \kappa}$.

Lemma 4.10 (i) For any $\varphi \in \mathcal{D}\left(G_{p}^{(1), \kappa}\right)$, one has $F(\varphi) \in \mathcal{F}$.

(ii) There exist functions $S(q)$ and $M\left(q, q^{\prime}\right)$ defined for $q, q^{\prime} \in G_{p}^{(1), \kappa}$, such that, for any $\varphi_{1}, \varphi_{2} \in \mathcal{D}\left(G_{p}^{(1), \kappa}\right)$, the following representation holds:

$$
\begin{array}{r}
\left(F\left(\varphi_{1}\right), F\left(\varphi_{2}\right)\right)_{\mathcal{F}}=\int_{\lambda_{1}(p)}^{\kappa} d x\left[\int_{\chi_{x}}\left(1+S_{x}(q)\right)\left|\varphi_{x}(q)\right|^{2} d \nu_{x}\right. \\
\left.+\int_{\chi_{x}} \int_{\chi_{x}} \bar{\varphi}_{x}(q) M_{x}\left(q, q^{\prime}\right) \varphi_{x}\left(q^{\prime}\right) d \nu_{x}(q) d \nu_{x}\left(q^{\prime}\right)\right] .
\end{array}
$$


Here, $\varphi_{x}, S_{x}$ and $M_{x}$ denote the restrictions of the functions $\varphi, S$ and $M$ to $\chi_{x}$ and $\chi_{x} \times \chi_{x}$, respectively.

(iii) The following estimates hold with suitable constants $\bar{C}, \hat{C}$ :

$$
\begin{gathered}
|S(q)| \leq \bar{C} \frac{\alpha}{\lambda_{2}(p)-\kappa}, \\
\left|M\left(q, q^{\prime}\right)\right| \leq \hat{C} \alpha h(q) h\left(q^{\prime}\right),
\end{gathered}
$$

implying that $F(\varphi) \in \mathcal{F}$, for $\varphi \in L^{2}\left(G_{p}^{(1), \kappa}\right)$ and

$$
C_{1}\|\varphi\|_{L^{2}\left(G_{p}^{(1), \kappa}\right)} \leq\|F(\varphi)\| \leq C_{2}\|\varphi\|_{L^{2}\left(G_{p}^{(1), \kappa}\right)}
$$

Proof: (i) This assertion will follow from the calculations below.

(ii) In the sense of distributions, equation (4.66) means that

$$
\left(F_{\xi(q)}^{q}, F_{\xi\left(q^{\prime}\right)}^{q^{\prime}}\right)_{\mathcal{F}}=(1+S(q)) \delta\left(q-q^{\prime}\right)+M\left(q, q^{\prime}\right) \delta\left(\xi(q)-\xi\left(q^{\prime}\right)\right)
$$

Before we proceed, the following remarks are in order:

$1^{\circ}$. We seemingly make an abuse in calculating the scalar product $\left(F_{\xi}^{q}, F_{\xi^{\prime}}^{q^{\prime}}\right)_{\mathcal{F}}$ of two generalized functions. Such calculations can be justified in the following way. The Fourier transform $\tilde{F}_{\xi, n}^{q}\left(\zeta_{1}, \ldots, \zeta_{n}\right)$ of the generalized function $F_{\xi, n}^{q}\left(q_{1}, \ldots, q_{n}\right)$ is, as one can easily verify, a usual function of the variables $\left(\zeta_{1}, \ldots, \zeta_{n}\right)$ polynomially bounded at infinity in these variables. If, further, we view the scalar product $\left(F_{\xi, n}^{q}, F_{\xi^{\prime}, n}^{q^{\prime}}\right)_{L^{2}\left(\mathbb{R}^{n d}\right)}$ as the limit of scalar products:

$$
\left(\tilde{F}_{\xi, n}^{q}, \tilde{F}_{\xi^{\prime}, n}^{q^{\prime}}\right)_{L^{2}\left(\mathbb{R}^{n d}\right)}:=\lim _{\delta \searrow 0} \int_{\mathbb{R}^{n d}} \overline{\tilde{F}_{\xi, n}^{q}\left(\zeta_{1}, \ldots, \zeta_{n}\right)} \tilde{F}_{\xi^{\prime}, n}^{q^{\prime}}\left(\zeta_{1}, \ldots, \zeta_{n}\right) \prod_{i=1}^{n}\left(e^{-\delta\left|\zeta_{i}\right|} d \zeta_{i}\right)
$$

then one can prove that this limit exists in the sense of convergence of generalized functions of the variables $q, q^{\prime}$. We shall not provide the details of this justifying procedure, and write instead directly its result entering our calculations.

$2^{\circ}$. We shall exploit the "orthogonality" of the generalized eigenfunctions $F_{\xi(q)}^{q}, q \in G_{p}^{(1), \kappa}$, corresponding to different eigenvalues $\xi(q) \neq \xi\left(q^{\prime}\right)$, by supposing that the support of the generalized function

$$
Q\left(q, q^{\prime}\right):=\left(F_{\xi(q)}^{q}, F_{\xi\left(q^{\prime}\right)}^{q^{\prime}}\right)_{\mathcal{F}}
$$

is contained in the surface $\Sigma=\left\{\left(q, q^{\prime}\right) \in G_{p}^{(1), \kappa} \times G_{p}^{(1), \kappa}: \xi(q)=\xi\left(q^{\prime}\right)\right\}$ :

$$
\operatorname{supp} Q \subset \Sigma
$$

and neglect in our calculation all terms which do not contribute to the factors in front of $\delta\left(\xi(q)-\xi\left(q^{\prime}\right)\right)$ or $\delta\left(q-q^{\prime}\right)$. However, the relation (4.71) likewise needs a justification. 
Namely, if we did not skip these "non-contributing" terms in the calculations, we would obtain a generalized function $\tilde{Q}\left(q, q^{\prime}\right)$, such that, for smooth functions $\varphi_{i}(q), i=1,2$ with support contained in $G_{p}^{(1), \kappa}$, the scalar product

$$
\left(F\left(\varphi_{1}\right), F\left(\varphi_{2}\right)\right)_{\mathcal{F}}=\int_{G_{p}^{(1), \kappa} \times G_{p}^{(1), \kappa}} \tilde{Q}\left(q, q^{\prime}\right) \bar{\varphi}_{1}(q) \varphi_{2}\left(q^{\prime}\right) d q d q^{\prime}
$$

would be finite, in particular, $F(\varphi) \in \mathcal{F}$ for smooth $\varphi$. On the other hand, if the support of $\varphi$ was contained in an $\varepsilon$-neighbourhood of the level $\chi_{x}$, then $F(\varphi) \in E(x-\varepsilon, x+\varepsilon) \mathcal{F}$, where $\{E(\Delta)\}$ denotes the family of spectral projections of $H_{p}$. Hence, for $\varphi_{i}(q), i=1,2$ with supports respectively contained in nonintersecting $\varepsilon$-neighbourhoods of the levels $\chi_{x_{i}}$, where $x_{1} \neq x_{2}$, the vectors $F\left(\varphi_{i}\right), i=1,2$, would be orthogonal. This proves in fact (4.711).

Keeping these remarks in mind, we proceed with the proof of (ii).

One has

$$
\left(F_{\xi(q)}^{q}, F_{\xi\left(q^{\prime}\right)}^{q^{\prime}}\right)_{\mathcal{F}}=\left(\Pi_{1} F_{\xi(q)}^{q}, \Pi_{1} F_{\xi\left(q^{\prime}\right)}^{q^{\prime}}\right)_{\mathcal{H}^{(\leq 1)}}+\sum_{n=2}^{\infty}\left(F_{\xi(q), n}^{q}, F_{\xi\left(q^{\prime}\right), n}^{q^{\prime}}\right)_{\mathcal{H}^{(n)}}
$$

As $H_{p}$ is self-adjoint and $F_{\xi(q)}^{q}$ are its generalized eigenfunctions with eigenvalue $\xi(q)$, the support of this distribution is contained in $\xi(q)=\xi\left(q^{\prime}\right)$. As $\Pi_{1} F_{\xi(q)}^{q}=\psi^{q}$ and $\Pi_{1} F_{\xi\left(q^{\prime}\right)}^{q^{\prime}}=$ $\psi^{q^{\prime}}$ are generalized eigenvectors of the operator $A(\xi)$ for $\xi=\xi(q)=\xi\left(q^{\prime}\right)$, we can use the relation (4.30):

$$
\left(\Pi_{1} F_{\xi(q)}^{q}, \Pi_{1} F_{\xi\left(q^{\prime}\right)}^{q^{\prime}}\right)_{\mathcal{H}(\leq 1)}=\delta\left(q-q^{\prime}\right),\left(\xi(q)=\xi\left(q^{\prime}\right)\right)
$$

We are therefore left with calculating $\left(F_{\xi(q), n}^{q}, F_{\xi\left(q^{\prime}\right), n}^{q^{\prime}}\right)_{\mathcal{H}^{(n)}}$ for $n \geq 2$. To this aim, use is made of the representation (3.9)

$$
\begin{aligned}
F_{\xi(q), n}^{q}= & \sum_{i=1}^{n} b_{n}\left(\xi(q) ; q_{1}, \ldots, \check{q}_{i} . ., q_{n} ; q_{i}\right) f_{\xi(q), 1}^{q}\left(q_{i}\right) \\
& +\int d_{n}\left(\xi(q) ; q_{1}, \ldots, q_{n} ; q^{\prime}\right) f_{\xi(q), 1}^{q}\left(q^{\prime}\right) d q^{\prime}
\end{aligned}
$$

The second term in (4.74) is a smooth function of $q_{1}, \ldots, q_{n}$ and does not contribute to the terms containing $\delta\left(\xi(q)-\xi\left(q^{\prime}\right)\right)$ or $\delta\left(q-q^{\prime}\right)$. Likewise, it is not hard to see that the only contributions to such terms come from

$$
\begin{array}{r}
\int \overline{b_{n}\left(\xi(q) ; q_{1} \ldots \check{q}_{i} \ldots q_{n} ; q_{i}\right) f_{\xi(q), 1}^{q}\left(q_{i}\right)} b_{n}\left(\xi\left(q^{\prime}\right) ; q_{1} \ldots \check{q}_{i} \ldots q_{n} ; q_{i}\right) f_{\xi\left(q^{\prime}\right), 1}^{q^{\prime}}\left(q_{i}\right) d q_{1} \ldots d q_{n} \\
=\int g_{n}\left(\xi(q), \xi\left(q^{\prime}\right) ; \hat{q}\right) \overline{f_{\xi(q), 1}^{q}(\hat{q})} f_{\xi\left(q^{\prime}\right), 1}^{q^{\prime}}(\hat{q}) d \hat{q},
\end{array}
$$

where

$$
g_{n}\left(\xi, \xi^{\prime} ; \hat{q}\right)=\int \overline{b_{n}\left(\xi ; q_{1} \ldots q_{n-1} ; \hat{q}\right)} b_{n}\left(\xi^{\prime} ; q_{1} \ldots q_{n-1} ; \hat{q}\right) d q_{1} \ldots d q_{n-1}
$$


In the integral over $\hat{q}$ in the r.h.s. of equation (4.75), we separate the singular parts of $f_{\xi(q), 1}^{q}, f_{\xi\left(q^{\prime}\right), 1}^{q^{\prime}}$ using the Sokhotski formula:

$$
\frac{1}{x+i 0}=\mathcal{P}\left(\frac{1}{x}\right)+i \pi \delta(x)
$$

in their expression (4.26) and the fact that $a_{p}\left(\xi(q), q^{\prime}\right)=\xi(q)$ implies $\xi(q)=\xi\left(q^{\prime}\right)$, hence also $a_{p}\left(\xi(q), q^{\prime}\right)=\xi\left(q^{\prime}\right)$ (in view of the uniqueness of the solution of $a_{p}(\xi, q)=\xi$ ):

$$
\begin{array}{r}
f_{\xi(q), 1}^{q}\left(q^{\prime}\right)=\delta\left(q-q^{\prime}\right)+i \pi R_{\xi(q)}\left(q, q^{\prime}\right) \\
\delta\left(\xi(q)-\xi\left(q^{\prime}\right)\right) \\
+ \text { regular terms }
\end{array}
$$

where

$$
\begin{aligned}
& R_{\xi}\left(q, q^{\prime}\right)=\alpha^{2} K_{\xi}^{-}\left(\xi ; q, q^{\prime}\right)-\alpha f_{\xi, 0}^{q} c(p-q, q) \\
& +\alpha^{2} f_{\xi, 0}^{q} \int K_{\xi}^{-}\left(\xi ; q^{\prime}, q^{\prime \prime}\right) c\left(p-q^{\prime \prime}, q^{\prime \prime}\right)\left(a_{p}\left(\xi, q^{\prime \prime}\right)-\xi+i 0\right)^{-1} d q^{\prime \prime} .
\end{aligned}
$$

The regular parts do not contribute to (4.75), which becomes, after performing the integration over $\hat{q}$ :

$$
\begin{aligned}
& g_{n}(\xi(q), \xi(q) ; q) \delta\left(q-q^{\prime}\right) \\
& +i \pi \delta\left(\xi(q)-\xi\left(q^{\prime}\right)\right)\left[g_{n}\left(\xi(q), \xi(q) ; q^{\prime}\right) R_{\xi(q)}\left(q, q^{\prime}\right)\right. \\
& \left.\quad-g_{n}(\xi(q), \xi(q) ; q) \bar{R}_{\xi(q)}\left(q^{\prime}, q\right)\right] \\
& +\pi^{2} \int_{\xi\left(q^{\prime \prime}\right)=\xi(q)} g_{n}\left(\xi(q), \xi(q) ; q^{\prime \prime}\right) R_{\xi(q)}\left(q, q^{\prime \prime}\right) \bar{R}_{\xi(q)}\left(q^{\prime}, q^{\prime \prime}\right) d q^{\prime \prime}
\end{aligned}
$$

Let us define the function:

$$
T\left(\xi, q^{\prime}\right)=\sum_{n=2}^{\infty} \frac{1}{n !} n g_{n}\left(\xi, \xi ; q^{\prime}\right)
$$

Then, one can see from equations (4.73), (4.79) that (4.70) is fulfilled with

$$
\begin{gathered}
S(q)=T(\xi(q), q), \\
M\left(q, q^{\prime}\right)=i \pi\left[T\left(\xi(q), q^{\prime}\right) R_{\xi(q)}\left(q, q^{\prime}\right)-T(\xi(q), q) \bar{R}_{\xi(q)}\left(q^{\prime}, q\right)\right] \\
+\pi^{2} \int T\left(\xi(q), q^{\prime \prime}\right) R_{\xi(q)}\left(q, q^{\prime \prime}\right) \bar{R}_{\xi(q)}\left(q^{\prime}, q^{\prime \prime}\right) d q^{\prime \prime} .
\end{gathered}
$$

(iii) Using the estimates in Lemma 3.2, one obtains for $T$ :

$$
\left|T\left(\xi, q^{\prime}\right)\right| \leq C \frac{\alpha}{\lambda_{2}(p)-\kappa} \sum_{n=2}^{\infty} \frac{\|h\|_{L_{2}}^{2(n-1)}}{(n-1) !}=\bar{C} \frac{\alpha}{\lambda_{2}(p)-\kappa} .
$$

Also, from the inequalities (4.51) and (4.8) it follows that

$$
\left|R_{\xi(q)}\left(q, q^{\prime}\right)\right| \leq C h(q) h\left(q^{\prime}\right),
$$


wherefrom (4.67), (4.68) follow. Using these estimates in equation (4.66), one obtains that $F(\varphi) \in \mathcal{F}$ for any $\varphi \in L^{2}\left(G_{p}^{(1), \kappa}\right)$ and, moreover the estimate (4.69) holds. The lemma is proved.

Let now $\mathcal{H}_{1}^{\kappa}(p) \subset \mathcal{F}$ be the subspace spanned by $\left\{F(\varphi), \varphi \in L^{2}\left(G_{p}^{(1), \kappa}\right)\right\}$. Equation (4.69) implies that the application $\varphi \longmapsto F(\varphi)$ is continuous and invertible. Thereby, $\mathcal{H}_{1}^{\kappa}(p)$ is $H_{p}$-invariant and

$$
H_{p} F(\varphi)=F(\hat{\xi} \varphi)
$$

where

$$
(\hat{\xi} \varphi)(q)=\xi(q) \varphi(q)
$$

Lemma 4.11 There exists a bounded, invertible operator $B: L^{2}\left(G_{p}^{(1), \kappa}\right) \rightarrow L^{2}\left(G_{p}^{(1), \kappa}\right)$ which commutes with $H_{p}$ and such that:

$$
\left(F\left(B \varphi_{1}\right), F\left(B \varphi_{2}\right)\right)_{\mathcal{F}}=\left(\varphi_{1}, \varphi_{2}\right)_{L^{2}\left(G_{p}^{(1), \kappa}\right)} \cdot
$$

Proof: We use the representation (4.65) of $L^{2}\left(G_{p}^{(1), \kappa}, d q\right)$ as a direct integral of the spaces $\mathcal{H}_{x}$ and write $\mathcal{H}_{1}^{\kappa}(p)$ as a direct integral:

$$
\mathcal{H}_{1}^{\kappa}(p)=\int_{\left[\lambda_{1}(p), \kappa\right]}^{\oplus} \mathcal{H}_{1, x} d x .
$$

Here $\mathcal{H}_{1, x}$ is the image of $\mathcal{H}_{x}$ by the application of $L^{2}\left(G_{p}^{(1), \kappa}, d q\right)$ into $\mathcal{H}_{1}^{\kappa}(p)$ and consists of functionals

$$
F_{x}(\varphi)=\int_{\chi_{x}} F_{\xi(q)}^{q} \varphi(q) d \nu_{x}(q) .
$$

By virtue of (4.70),

$$
\begin{array}{r}
\left(F_{x}\left(\varphi_{1}\right), F_{x}\left(\varphi_{2}\right)\right)_{\mathcal{F}}=\int_{\chi_{x}}(1+S(q)) \bar{\varphi}_{1}(q) \varphi_{2}(q) d \nu_{x}(q) \\
+\int_{\chi_{x} \times \chi_{x}} \bar{\varphi}_{1}(q) M\left(q, q^{\prime}\right) \varphi_{2}\left(q^{\prime}\right) d \nu_{x}(q) d \nu_{x}\left(q^{\prime}\right) \\
=\left(\left(I_{x}+V_{x}\right) \varphi_{1}, \varphi_{2}\right)_{\mathcal{H}_{1, x}}
\end{array}
$$

where $I_{x}$ is the unit operator in $\mathcal{H}_{1, x}$ and $V_{x}$ is a bounded operator with small norm (cf. equations (4.67), (4.68) ). Also, $H_{p}$ acts in $\mathcal{H}_{1, x}$ as $x I_{x}$.

Let $B_{x}=\left(I_{x}+V_{x}\right)^{-1 / 2}$. Then, equation (4.88) reads as

$$
\left(F_{x}\left(B_{x} \varphi_{1}\right), F_{x}\left(B_{x} \varphi_{2}\right)\right)_{\mathcal{F}}=\left(\varphi_{1}, \varphi_{2}\right)_{\mathcal{H}_{1, x}} .
$$

Finally, defining $B=\int_{\left[\lambda_{1}(p), \kappa\right]}^{\oplus} B_{x} d x$, one gets both that the operator $B$ commutes with $H_{p}$ and that equation (4.85) is satisfied . 


\section{The ground state of $H_{p}$}

A detailed analysis of the ground state of $H_{p}$ is performed in arbitrary dimension in [2]. In this section we shall briefly show how the existence of the ground state follows from our considerations for $d \geq 3$.

As explained in Section 2, $H_{p}$ has a ground state if, and only if, there exists $\xi<\lambda_{1}(p)$, such that operator $A_{p}(\xi)$ in $\mathcal{H}^{(\leq 1)}$ has the eigenvalue $\xi$. By the analysis done in Section 4.1. $A_{p}(\xi)$ has one simple eigenvalue $e_{p}(\xi)<\lambda_{1}(p)$ if, and only if, $\Delta_{p}\left(\lambda_{1}(p)\right)<0$ (where $\Delta_{p}(\xi)$ is the function defined by equation (4.21) for $A_{p}(\xi)$ ), in which case $e_{p}(\xi)$ equals the unique solution of the equation $\Delta_{p}(\xi)=0$. Since $e_{p}^{(0)}-\lambda_{1}(p) \rightarrow \infty$ for $p \rightarrow \infty$, while $\left(v, R_{B}\left(\lambda_{1}(p)\right) v\right)$ (with $v$ and $B$ corresponding to $\left.A_{p}(\xi)\right)$ is bounded, $\left\{p ; \Delta_{p}\left(\lambda_{1}(p)\right)<0\right\}$ is a bounded domain.

As seen from equation (2.10), $A_{p}(\xi)$ is a decreasing family (in the usual order of selfadjoint operators), implying that $e_{p}(\xi)$ is a decreasing function of $\xi \in\left(-\infty, \lambda_{1}(p)\right)$. We conclude that the equation $e_{p}(\xi)=\xi$ has a solution $\xi_{p}^{(0)}$ if, and only if, $p$ belongs to the subdomain

$$
G^{(0)}=\left\{p: e_{p}\left(\lambda_{1}(p)\right)<\lambda_{1}(p)\right\}
$$

For $p \in G^{(0)}$, let $F_{\xi_{p}^{(0)}, 1}$ be an eigenvector of $A_{p}\left(\xi_{p}^{(0)}\right)$, and $F_{\xi_{p}, 2} \in \mathcal{H}^{(\geq 2)}$ be defined according to (2.14) . Then, the vector $F_{p}^{(0)}=F_{\xi_{p}^{(0)}, 1}+F_{\xi_{p}^{(0)}, 2}$ is a ground state of the operator $H_{p}$.

Therefore, $H_{p}$ has a unique ground state if $p \in G^{(0)}$, and no ground state if $p \notin G^{(0)}$.

\section{Concluding remarks}

The main result of the paper is the construction, in the weak coupling regime, of a manifold of states indexed by a phonon momentum $q$. The ground state describing a single polaron becomes unstable at a certain momentum threshold, above which it dissolves into this manifold. It is to be expected that at still higher momenta the latter states become themselves unstable and dissolve into two-phonon states, etc. The representation (4.56) of the eigenvalue $\xi(q)$ strongly suggests the interpretation of the generalized eigenfunctions $F_{\xi(q)}^{q}$ associated to it as scattering states of a free phonon and a certain particle with the dispersion law $\gamma(k)$. We cannot yet decide whether the latter particle is a polaron defined in our Theorem 1.1] i.e. whether $\gamma(k)=\xi_{k}^{(0)}$, although we checked that this equality is true in the first nontrivial order in coupling constant: $\sim \alpha^{2}$. In this case the ground state instability at high $k$ might be interpreted as emission of a phonon.

Unfortunately, we were not able to prove in the present paper two essential results in favour of the above heuristic physical picture:

1. First of all, we did not construct the whole one-boson subspace $\mathcal{H}_{1}^{\kappa=\lambda_{2}(p)}$ up to the two-boson threshold $\lambda_{2}(p)$. The approach used here of eliminating the higher components of the eigenvectors can equally well be applied in the case of the decomposition $\mathcal{F}=$ $\mathcal{H}^{\leq 2} \oplus \mathcal{H}^{\geq 3}$, leading to a family of self-adjoint operators $\left\{A(\xi), \lambda_{1}(p) \leq \xi \leq \kappa\right\}$, (where 
$\lambda_{2}(p) \leq \kappa<\lambda_{3}(p)$, i.e. $\kappa$ is between the two-boson and the three-boson threshold, defined similarly with $\left.\lambda_{2}(p)\right)$, acting in the space $\mathcal{H} \leq 2$ of triples $\left(f_{0}, f_{1}(\cdot), f_{2}(\cdot, \cdot)\right)$. These operators have a more complicated structure than the Friedrichs operators in $\mathcal{H}^{\leq 1}$ and their spectral analysis and scattering theory is not available in such details as for the Friedrichs operators. If this theory was elaborated (e.g. using equations analogous to the Faddeev-Yakubovski equations for the resolvent of $n$-body Schrödinger operators, see [1] - [13]), then the approach of the present paper would provide the construction of the whole one-boson subspace and of a part of the two-boson subspace.

2. Secondly, we did not prove the completeness of the constructed subspaces $\mathcal{H}_{0}(p)$ (generated by the ground state) and $\mathcal{H}_{1}^{\kappa}(p)$, meaning that in $\left(\mathcal{H}_{0}(p) \oplus \mathcal{H}_{1}^{\kappa}(p)\right)^{\perp}$ the spectrum of $H_{p}$ has no point below $\kappa$. We are convinced that this assertion is true and hope to prove it in the future.

\section{Acknowledgements}

N.A. and R.A.M. acknowledge the warm hospitality of the C.P.T. Luminy-Marseille, where the project of this work was born. R.A.M. also acknowledges financial support from the Scientific Fund of the Russian Federation (Grant No. 02-01-00444) and the Presidential Fund for Support of Scientific Schools of Russia (Grant No. 934 2003.1).

\section{References}

[1] H.Fröhlich, Electrons in lattice fields, Adv.Phys. 3, 325-364 (1954).

[2] R.A.Minlos, Lower branch of the spectrum of a fermion interacting with a bosonic gas (polaron), Theor. Math. Phys. 92, 255-268 (1992).

[3] H.Spohn, The polaron at large total momentum, J.Phys.A 21, 1199-1212 (1988).

[4] B.Gerlach, H.Löwen, Analytical properties of polaron systems or: Do polaronic phase transitions exist or not?, Rev.Mod.Phys. 63, 63-90 (1991).

[5] J.Fröhlich, Existence of dressed one electron states in a class of persistent models, Fortschr.Phys. 22, 159-198 (1974).

[6] K.O.Friedrichs, Perturbation of Spectra in Hilbert Space, Amer. Math. Soc., Providence, 1965.

[7] D.R.Yafaev, Scattering Theory, St.-Petersburg University, 1994.

[8] M.Reed and B.Simon, Methods of Modern Mathematical Physics Vol. IV, Academic Press, New York, 1983.

[9] I.M.Gelfand and G.E.Shilov, Generalized Functions, Vol.3, Nauka, Moscow, 1959.

[10] Yu.M.Berezanski Eigenfunction Expansion of Self-Adjoint Operators, Naukova Dumka, Kiev, 1965. 
[11] O.A.Yakubovski, On the integral equations in the theory of N-particle scattering, Yadernaya Fizika 5, 1312-1320 (1967).

[12] L.D.Faddeev, Mathematical questions in the quantum theory of scattering for a system of three particles, Trudy Mat. Inst. Steklov. 69 122pp (1963)

[13] K.Hepp, On the quantum-mechanical N-body problem, Helv.Phys.Acta 42, 425-458 (1969). 